\title{
ON PRODUCTS OF QUASICONVEX SUBGROUPS IN HYPERBOLIC GROUPS
}

\author{
Ashot Minasyan
}

\begin{abstract}
An interesting question about quasiconvexity in a hyperbolic group concerns finding classes of quasiconvex subsets that are closed under finite intersections. A known example is the class of all quasiconvex subgroups [1]. However, not much is yet learned about the structure of arbitrary quasiconvex subsets. In this work we study the properties of products of quasiconvex subgroups; we show that such sets are quasiconvex, their finite intersections have a similar algebraic representation and, thus, are quasiconvex too.
\end{abstract}

\section{Introduction}

Let $G$ be a hyperbolic group, $\Gamma(G, \mathcal{A})$ - its Cayley graph corresponding to a finite symmetrized generating set $\mathcal{A}$ (i.e. for each element $a \in \mathcal{A}, a^{-1}$ also belongs to this set). A subset $Q \subseteq G$ is said to be $\varepsilon$-quasiconvex, if any geodesic connecting two elements from $Q$ belongs to a closed $\varepsilon$-neighborhood $\mathcal{O}_{\varepsilon}(Q)$ of $Q$ in $\Gamma(G, \mathcal{A})$ for some $\varepsilon \geq 0$. $Q$ will be called quasiconvex if there exists $\varepsilon>0$ for which it is $\varepsilon$-quasiconvex.

In [4] Gromov proves that the notion of quasiconvexity in a hyperbolic group does not depend on the choice of a finite generating set (it is easy to show that this is not true in an arbitrary group).

If $A, B \subseteq G$ then their product is a subset of $G$ defined by $A \cdot B=\{a b \mid a \in A, b \in B\}$.

Proposition 1. If the sets $A_{1}, \ldots, A_{n} \subset G$ are quasiconvex then their product set $A_{1} A_{2} \cdot \ldots \cdot A_{n} \stackrel{\text { def }}{=}\left\{a_{1} a_{2} \cdot \ldots \cdot a_{n} \mid a_{i} \in G_{i}\right\} \subset G$ is also quasiconvex.

Proposition 1 was proved by Zeph Grunschlag in 1999 in [11; Prop. 3.14] and, independently, by the author in his diploma paper in 2000 .

If $H$ is a subgroup of $G$ and $x \in G$ then the subgroup conjugated to $H$ by $x$ will be denoted $H^{x}=x H x^{-1}$. The main result of the paper is

Theorem 1. Suppose $G_{1}, \ldots, G_{n}, H_{1}, \ldots, H_{m}$ are quasiconvex subgroups of the group $G, n, m \in \mathbb{N} ; f, e \in G$. Then there exist numbers $r, t_{l} \in \mathbb{N} \cup\{0\}$ and $f_{l}, \alpha_{l k}, \beta_{l k} \in G, k=1,2, \ldots, t_{l}$ (for every fixed $l$ ), $l=1,2, \ldots, r$, such that

$$
f G_{1} G_{2} \cdot \ldots \cdot G_{n} \cap e H_{1} H_{2} \cdot \ldots \cdot H_{m}=\bigcup_{l=1}^{r} f_{l} S_{l}
$$

where for each $l, t=t_{l}$, there are indices $1 \leq i_{1} \leq i_{2} \leq \ldots \leq i_{t} \leq n, 1 \leq j_{1} \leq$ $\leq j_{2} \leq \ldots \leq j_{t} \leq m$ :

$$
S_{l}=\left(G_{i_{1}}^{\alpha_{l 1}} \cap H_{j_{1}}^{\beta_{l 1}}\right) \cdot \ldots \cdot\left(G_{i_{t}}^{\alpha_{l t}} \cap H_{j_{t}}^{\beta_{l t}}\right) .
$$


This claim does not hold if the group $G$ is not hyperbolic : set $G_{1}=\langle(1,0)\rangle$, $G_{2}=\langle(0,1)\rangle, H=\langle(1,1)\rangle$ - cyclic subgroups of $\mathbb{Z}^{2}$ (they are quasiconvex in $\mathbb{Z}^{2}$ with generators $\{(1,0),(0,1),(1,1)\}) \cdot G_{1} \cdot G_{2}=\mathbb{Z}^{2}$, thus, $G_{1} G_{2} \cap H=H$ but $G_{1} \cap H=G_{2} \cap H=\{(0,0)\}$ and, if the statement of the theorem held for $\mathbb{Z}^{2}$ then $H$ would be finite - a contradiction.

The above example can also be used as another argument to prove the wellknown fact that $\mathbb{Z}^{2}$ can not be embedded into a hyperbolic group (because any cyclic subgroup is quasiconvex in a hyperbolic group).

The condition that the subgroups $G_{i}, H_{j}$ are quasiconvex is also necessary: using Rips' Construction ([12]) one can achieve a group $G$ satisfying the small cancellation condition $C^{\prime}(1 / 6)$ (and, therefore, hyperbolic) and its finitely generated normal subgroup $K$ such that $G / K \cong \mathbb{Z}^{2}$. Let $\phi$ be the natural epimorphism from $G$ to $\mathbb{Z}^{2}, G_{1}=\phi^{-1}(\langle(1,0)\rangle) \leq G, G_{2}=\phi^{-1}(\langle(0,1)\rangle) \leq G$, $H=\phi^{-1}(\langle(1,1)\rangle) \leq G$. $G_{1}, G_{2}$ and $H$ are finitely generated subgroups of $G$, $G_{1} \cdot G_{2}=G$ because $\langle(1,0)\rangle \cdot\langle(0,1)\rangle=\mathbb{Z}^{2}$ and $K \leq G_{2}$, thus $G_{1} \cdot G_{2} \cap H=H$. But for every $\alpha, \beta \in G \phi\left(G_{i}^{\alpha} \cap H^{\beta}\right) \subseteq \phi\left(G_{i}\right)^{\phi(\alpha)} \cap \phi(H)^{\phi(\beta)}=\{(0,0)\}, i=1,2$. Hence, it is impossible to obtain the infinite subgroup $\phi(H)$ from products of cosets to such sets, and we constructed the counterexample needed.

Definition : let $G_{1}, G_{2}, \ldots, G_{n}$ be quasiconvex subgroups of $G, f_{1}, f_{2}, \ldots$, $f_{n} \in G, n \in \mathbb{N}$. Then the set

$$
f_{1} G_{1} f_{2} G_{2} \cdot \ldots \cdot f_{n} G_{n}=\left\{f_{1} g_{1} f_{2} g_{2} \cdot \ldots \cdot f_{n} g_{n} \in G \mid g_{i} \in G_{i}, i=1, \ldots, n\right\}
$$

will be called quasiconvex product.

Corollary 2. An intersection of finitely many quasiconvex products is a finite union of quasiconvex products.

Thus the class of finite unions of quasiconvex products is closed under taking finite intersections.

Recall that a group $H$ is called elementary if it has a cyclic subgroup $\langle h\rangle$ of finite index. An elementary subgroup of a hyperbolic group is quasiconvex (see remark 5, Section 4). It is well known that any element $x$ of infinite order in $G$ is contained in a unique maximal elementary subgroup $E(x) \leqslant G[4]$, [5].

Every non-elementary hyperbolic group contains the free group of rank $2[5$, Cor. 6].

Suppose $G_{1}, G_{2}, \ldots, G_{n}, H_{1}, H_{2}, \ldots, H_{m}$ are infinite maximal elementary subgroups of $G, f, e \in G$. And $G_{i} \neq G_{i+1}, H_{j} \neq H_{j+1}, i=1, \ldots, n-1$, $j=1, \ldots, m-1$. Then we present the following uniqueness result for the products of such subgroups:

Theorem 2. The sets $f G_{1} \cdot \ldots \cdot G_{n}$ and $e H_{1} \cdot \ldots \cdot H_{m}$ are equal if and only if $n=m, G_{n}=H_{n}$, and there exist elements $z_{j} \in H_{j}, j=1, \ldots, n$, such that $G_{j}=\left(z_{n} z_{n-1} \ldots z_{j+1}\right) \cdot H_{j} \cdot\left(z_{n} z_{n-1} \ldots z_{j+1}\right)^{-1}, j=1,2, \ldots, n-1$, $f=e z_{1}^{-1} z_{2}^{-1} \ldots z_{n}^{-1}$.

Similarly to quasiconvex products one can define ME-products to be the products of cosets of maximal elementary subgroups in $G$ (the full definition is 
given in Section 4). The statement of Corollary 2 can be strengthened in this case :

Theorem 3. Intersection of any family (finite or infinite) of finite unions of ME-products is a finite union of ME-products.

An example which shows that an analogous property is not true for arbitrary quasiconvex products is constructed at the end of this paper.

Thus, all finite unions of ME-products constitute a topology $\mathcal{T}$ (of closed sets) on the set of elements of a hyperbolic group. Taking an inverse, left and right shifts in $G$ are continuous operations in $\mathcal{T}$. Also, by definition, any point is closed in $\mathcal{T}$, so $\mathcal{T}$ is weakly separated $\left(T_{1}\right)$. However, if $G$ is infinite elementary, then $\mathcal{T}$ turns out to be the topology of finite complements which is not Hausdorff, also, in this case, the group multiplication is not continuous with respect to $\mathcal{T}$ (since any product of two non-empty open sets contains the identity of $G$ ).

\section{Preliminary information}

Let $d(\cdot, \cdot)$ be the usual left-invariant metric on the Cayley graph of the group $G$ with generating set $\mathcal{A}$. For any two points $x, y \in \Gamma(G, \mathcal{A})$ we fix a geodesic path between them and denote it by $[x, y]$.

If $Q \subset \Gamma(G, \mathcal{A}), N \geq 0$, the closed $N$-neighborhood of $Q$ will be denoted by

$$
\mathcal{O}_{N}(Q) \stackrel{\text { def }}{=}\{x \in \Gamma(G, \mathcal{A}) \mid d(x, Q) \leq N\} .
$$

If $x, y, w \in \Gamma(G, \mathcal{A})$, then the number

$$
(x \mid y)_{w} \stackrel{\text { def }}{=} \frac{1}{2}(d(x, w)+d(y, w)-d(x, y))
$$

is called the Gromov product of $x$ and $y$ with respect to $w$.

Let $a b c$ be a geodesic triangle. There exist "special" points $O_{a} \in[b, c]$, $O_{b} \in[a, c], O_{c} \in[a, b]$ with the properties: $d\left(a, O_{b}\right)=d\left(a, O_{c}\right)=\alpha, d\left(b, O_{a}\right)=$ $=d\left(b, O_{c}\right)=\beta, d\left(c, O_{a}\right)=d\left(c, O_{b}\right)=\gamma$. From a corresponding system of linear equations one can find that $\alpha=(b \mid c)_{a}, \beta=(a \mid c)_{b}, \gamma=(a \mid b)_{c}$. Two points $O \in[a, b]$ and $O^{\prime} \in[a, c]$ are called $a$-equidistant if $d(a, O)=d\left(a, O^{\prime}\right) \leq \alpha$.

The triangle $a b c$ is said to be $\delta$-thin if for any two points $O, O^{\prime}$ lying on its sides and equidistant from one of its vertices, $d\left(O, O^{\prime}\right) \leq \delta$ holds.

$a b c$ is $\delta$-slim if each of its sides belongs to a closed $\delta$-neighborhood of the two others.

We assume the following equivalent definitions of hyperbolicity of $\Gamma(G, \mathcal{A})$ to be known to the reader (see [6], [2]):

$1^{\circ}$. There exists $\delta \geq 0$ such that for any four points $x, y, z, w \in \Gamma(G, \mathcal{A})$ their Gromov products satisfy

$$
(x \mid y)_{w} \geq \min \left\{(x \mid z)_{w},(y \mid z)_{w}\right\}-\delta ;
$$

$2^{\circ}$. All triangles in $\Gamma(G, \mathcal{A})$ are $\delta$-thin for some $\delta \geq 0$;

$3^{\circ}$. All triangles in $\Gamma(G, \mathcal{A})$ are $\delta$-slim for some $\delta \geq 0$. 
Now and below we suppose that $G$ meets $1^{\circ}, 2^{\circ}$ and $3^{\circ}$ for a fixed (sufficiently large) $\delta \geq 0.3^{\circ}$ easily implies

Remark 0. Any side of a geodesic $n$-gon $(n \geq 3)$ in $\Gamma(G, \mathcal{A})$ belongs to a closed $(n-2) \delta$-neighborhood of the union of the rest of its sides.

Let $p$ be a path in the Cayley graph of $G$. Further on by $p_{-}, p_{+}$we will denote the startpoint and the endpoint of $p$, by $\|p\|$ - its length; lab $(p)$, as usual, will mean the word in the alphabet $\mathcal{A}$ written on $p \operatorname{elem}(p) \in G$ will denote the element of the group $G$ represented by the word $l a b(p)$.

A path $q$ is called $(\lambda, c)$-quasigeodesic if there exist $0<\lambda \leq 1, c \geq 0$, such that for any subpath $p$ of $q$ the inequality $\lambda\|p\|-c \leq d\left(p_{-}, p_{+}\right)$holds.

In a hyperbolic space quasigeodesics and geodesics with same ends are mutually close :

Lemma 1.1. $([6 ; 5.6,5.11],[2 ; 3.3])$ There is a constant $N=N(\delta, \lambda, c)$ such that for any $(\lambda, c)$-quasigeodesic path $p$ in $\Gamma(G, \mathcal{A})$ and a geodesic $q$ with $p_{-}=q_{-}, p_{+}=q_{+}$, one has $p \subset \mathcal{O}_{N}(q)$ and $q \subset \mathcal{O}_{N}(p)$.

An important property of cyclic subgroups in a hyperbolic group states

Lemma 1.2. $([6 ; 8.21],[2 ; 3.2])$ For any word $w$ representing an element $g \in G$ of infinite order there exist constants $\lambda>0, c \geq 0$, such that any path with a label $w^{m}$ in the Cayley graph of $G$ is $(\lambda, c)$-quasigeodesic for arbitrary integer $m$.

A broken line $p=\left[X_{0}, X_{1}, \ldots, X_{n}\right]$ is a path obtained as a consequent concatenation of geodesic segments $\left[X_{i-1}, X_{i}\right], i=1,2, \ldots, n$. Later, in this paper, we will use the following fact concerning broken lines in a hyperbolic space:

Lemma 1.3. $\left(\left[3\right.\right.$, Lemma 21]) Let $p=\left[X_{0}, X_{1}, \ldots, X_{n}\right]$ be a broken line in $\Gamma(G, \mathcal{A})$ such that $\left\|\left[X_{i-1}, X_{i}\right]\right\|>C_{1} \forall i=1, \ldots, n$, and $\left(X_{i-1} \mid X_{i+1}\right)_{X_{i}} \leq C_{0}$ $\forall i=1, \ldots, n-1$, where $C_{0} \geq 14 \delta, C_{1}>12\left(C_{0}+\delta\right)$. Then $p$ is contained in the closed $2 C_{0}$-neighborhood $\mathcal{O}_{2 C_{0}}\left(\left[X_{0}, X_{n}\right]\right)$ of the geodesic segment $\left[X_{0}, X_{n}\right]$.

Suppose $H=\langle\mathcal{X}\rangle$ is a subgroup of $G$ with a finite symmetrized generating set $\mathcal{X}$. If $h \in H$, then by $|h|_{G}$ and $|h|_{H}$ we will denote the lengths of the element $h$ in $\mathcal{A}$ and $\mathcal{X}$ respectively. The distortion function $D_{H}: \mathbb{N} \rightarrow \mathbb{N}$ of $H$ in $G$ is defined by $D_{H}(n)=\max \left\{\left.|h|_{H}|h \in H| h\right|_{G} \leq n,\right\}$.

If $\alpha, \beta: \mathbb{N} \rightarrow \mathbb{N}$ are two functions then we write $\alpha \preceq \beta$ if $\exists K_{1}, K_{2}>0$ : $\alpha(n) \leq K_{1} \beta\left(K_{2} n\right)$. $\alpha(n)$ and $\beta(n)$ are said to be equivalent if $\alpha \preceq \beta$ and $\beta \preceq \alpha$.

Evidently, the function $D_{H}$ does not depend (up to this equivalence) on the choice of finite generating sets $\mathcal{A}$ of $G$ and $\mathcal{X}$ of $H$. One can also notice that $D_{H}(n)$ is always at least linear (provided that $H$ is infinite). If $D_{H}$ is equivalent to linear, $H$ is called undistorted.

Lemma 1.4. ([2; 3.8], $[7 ; 10.4 .2])$ A quasiconvex subgroup $H$ of a hyperbolic group $G$ is finitely generated.

Remark 1. From the proof of this statement it also follows that $D_{H}$ is equivalent to linear for a quasiconvex subgroup $H$.

Indeed, it was observed in [2] that if $H$ is $\varepsilon$-quasiconvex, it is generated by 
finitely many elements $x_{i}, i=1, \ldots, s$, such that $\left|x_{i}\right|_{G} \leq 2 \varepsilon+1 \forall i$, and $\forall h \in H$, $h=a_{1} \cdot \ldots \cdot a_{r}, a_{j} \in \mathcal{A}$, hence $\exists i_{1}, \ldots, i_{r} \in\{1,2, \ldots, s\}: h=x_{i_{1}} x_{i_{2}} \cdot \ldots \cdot x_{i_{r}}$.

The proof of corollary 2 is based on

Lemma 1.5. ([1; Prop. 3]) Let $G$ be a group generated by a finite set $\mathcal{A}$. Let $A, B$ be subgroups of $G$ quasiconvex with respect to $\mathcal{A}$. Then $A \cap B$ is quasiconvex with respect to $\mathcal{A}$.

We will use the following notion in this paper :

Definition : let $H=\langle\mathcal{X}\rangle \leq G=\langle\mathcal{A}\rangle, \operatorname{card}(\mathcal{X})<\infty, \operatorname{card}(\mathcal{A})<\infty$. A path $P$ in $\Gamma(G, \mathcal{A})$ will be called $H$-geodesic (or just $H$-path) if :

a) $P$ is labelled by the word $a_{11} \ldots a_{1 k_{1}} \ldots a_{s 1} \ldots a_{s k_{s}}$ corresponding to an element $\operatorname{elem}(P)=x \in H$, where $a_{i j} \in \mathcal{A}$;

b) $a_{j 1} \ldots a_{j k_{j}}$ is a shortest word for generator $x_{j} \in \mathcal{X}$ (i.e. $\left|x_{j}\right|_{G}=k_{j}$ ), $j=1, \ldots, s$;

c) $x=x_{1} \ldots x_{s}$ in $H,|x|_{H}=s$.

I.e. $P$ is a broken line in $\Gamma(G, \mathcal{A})$ with segments corresponding to shortest representations of generators of $H$ by means of $\mathcal{A}$.

Lemma 1.6. (see also [10; Lemma 2.4]) Let $H$ be a (finitely generated) subgroup of a $\delta$-hyperbolic group $G$. Then $H$ is quasiconvex iff $H$ is undistorted in $G$.

Proof. The necessity is given by remark 1 .

To prove the sufficiency, suppose $H=\langle\mathcal{X}\rangle, \operatorname{card}(\mathcal{X})<\infty$, and $D_{H}(n) \leq c n$, $\forall n \in \mathbb{N}, c>0$. For arbitrary two vertices $x, y \in H$ there is a $H$-path $q$ connecting them in $\Gamma(G, \mathcal{A})$. Let $p$ be any its subpath. By definition, there exists a subpath $p^{\prime}$ of $q$ such that $p_{-}^{\prime}, p_{+}^{\prime} \in H$, subpaths of $q$ from $p_{-}$to $p_{-}^{\prime}$ and from $p_{+}$to $p_{+}^{\prime}$ are geodesic, and $d\left(p_{-}, p_{-}^{\prime}\right) \leq \varkappa / 2, d\left(p_{+}, p_{+}^{\prime}\right) \leq \varkappa / 2$, where $\varkappa=\max \left\{|h|_{G} \mid h \in \mathcal{X}\right\}<\infty$. In particular, $p^{\prime}$ is also $H$-geodesic.

Using the property c) from the definition of a $H$-path we obtain

$$
\left\|p^{\prime}\right\| \leq \varkappa \cdot\left|\operatorname{elem}\left(p^{\prime}\right)\right|_{H} \leq \varkappa \cdot c \cdot d\left(p_{-}^{\prime}, p_{+}^{\prime}\right) .
$$

Therefore, $\|p\| \leq\left\|p^{\prime}\right\|+\varkappa \leq \varkappa \cdot c \cdot d\left(p_{-}^{\prime}, p_{+}^{\prime}\right)+\varkappa \leq \varkappa \cdot c \cdot d\left(p_{-}, p_{+}\right)+\varkappa^{2} c+\varkappa$, which shows that $q$ is $\left(\frac{1}{\varkappa c}, \varkappa+\frac{1}{c}\right)$-quasigeodesic. By lemma $1.1 \exists N=N(\varkappa, c)$ such that any geodesic path between $x$ and $y$ belongs to the closed $N$-neighborhood $\mathcal{O}_{N}(q)$ but $q \subset \mathcal{O}_{\varkappa / 2}(H)$ in the Cayley graph of $G$. Hence, $H$ is quasiconvex with the constant $(N+\varkappa / 2)$, and the lemma is proved.

During this proof we showed

Remark 2. If $H$ is a quasiconvex subgroup of a hyperbolic group $G$ then any $H$-path is $(\lambda, c)$-quasigeodesic for some $\lambda, c$ depending only on the subgroup $H$.

Let the words $W_{1}, \ldots, W_{l}$ represent elements $w_{1}, \ldots, w_{l}$ of infinite order in a hyperbolic group $G$. For a fixed constant $K$ consider the set

$S_{M}=S\left(W_{1}, \ldots, W_{l} ; K, M\right)$ of words

$$
W=X_{0} W_{1}^{\alpha_{1}} X_{1} W_{2}^{\alpha_{2}} X_{2} \ldots W_{l}^{\alpha_{l}} X_{l}
$$


where ||$X_{i} \| \leq K$ for $i=0,1, \ldots, l,\left|\alpha_{2}\right|, \ldots,\left|\alpha_{l-1}\right| \geq M$, and the element of $G$ represented by $X_{i}^{-1} W_{i} X_{i}$ does not belong to the maximal elementary subgroup $E\left(w_{i+1}\right) \leq G$ containing $w_{i+1}$ for $i=1, \ldots, l-1$.

Lemma 1.7. ([5; Lemma 2.4]) There exist constants $\lambda>0, c \geq 0$ and $M>0$ (depending on $\left.K, W_{1}, \ldots, W_{l}\right)$ such that any path in $\Gamma(G, \mathcal{A})$ labelled by an arbitrary word $W \in S_{M}$ is $(\lambda, c)$-quasigeodesic.

Lemma 1.8. Suppose $l \in \mathbb{N}, K>0$, and $w_{1}, \ldots, w_{l} \in G$ are elements of infinite order. Then there are $\lambda>0, c \geq 0$ and $M>0$ (depending on $K$, $\left.w_{1}, \ldots, w_{l}\right)$ such that for arbitrary $x_{0}, x_{1}, \ldots, x_{l} \in G,\left|x_{i}\right|_{G} \leq K, i=0, \ldots, l$, with conditions $\quad w_{i} \notin x_{i} E\left(w_{i+1}\right) x_{i}^{-1} \forall i \in\{1, \ldots, l-1\}$, and any $\alpha_{i} \in \mathbb{Z}$, $\left|\alpha_{i}\right| \geq M, i=2, \ldots, l-1$, the element

$$
w=x_{0} w_{1}^{\alpha_{1}} x_{1} w_{2}^{\alpha_{2}} x_{2} \cdot \ldots \cdot w_{l}^{\alpha_{l}} x_{l} \in G
$$

satisfies $|w|_{G} \geq \lambda\left|\alpha_{1}\right|-c$.

Proof. As follows from Lemma 1.7 and the definition of a $(\lambda, c)$-quasigeodesic path, one has the following inequality:

$$
|w|_{G} \geq \lambda \cdot\left(\left|x_{0}\right|_{G}+\sum_{i=1}^{l}\left(\left|\alpha_{i}\right|\left|w_{i}\right|_{G}+\left|x_{i}\right|_{G}\right)\right)-c \geq \lambda \cdot\left|\alpha_{1}\right|\left|w_{1}\right|_{G}-c \geq \lambda\left|\alpha_{1}\right|-c .
$$

\section{Quasiconvex sets and their products}

Remark 3. Any finite subset of $G$ is $d$-quasiconvex (where $d$ is the diameter of this set).

Remark 4. Let $Q \subseteq G$ be $\varepsilon$-quasiconvex, $g \in G$. Then (a) the left shift $g Q=\{g x \mid x \in Q\}$ is quasiconvex with the same constant; (b) the right shift $Q g=\{x g \mid x \in Q\}$ is quasiconvex (possibly, with a different quasiconvexity constant).

(a) holds because the metric $d(\cdot, \cdot)$ is left-invariant.

$x, y \in Q$ if and only if $x g, y g \in Q g$. By remark 0

$$
\begin{aligned}
& {[x g, y g] \subset \mathcal{O}_{2 \delta}([x, x g] \cup[x, y]}\cup[y, y g]) \subset \mathcal{O}_{2 \delta+|g|_{G}}([x, y]) \subset \mathcal{O}_{2 \delta+|g|_{G}+\varepsilon}(Q) \subset \\
& \subset \mathcal{O}_{2 \delta+2|g|_{G}+\varepsilon}(Q g)
\end{aligned}
$$

therefore (b) is true.

Therefore, a left coset of a quasiconvex subgroup and a conjugate subgroup to it are quasiconvex (in a hyperbolic group).

Lemma 2.1. (see also [11; Prop. 3.14]) A finite union of quasiconvex sets in a hyperbolic group $G$ is quasiconvex.

Proof. It is enough to prove that if $A, B \subset G$ are $\varepsilon_{i}$-quasiconvex, $i=1,2$, respectively, then $C=A \cup B$ is quasiconvex. 


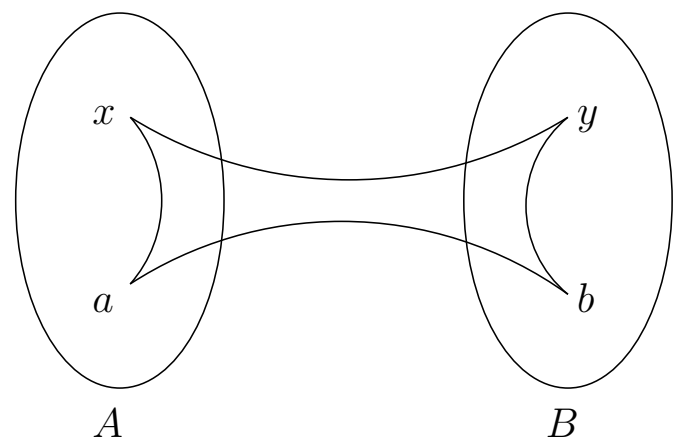

Figure 1

If both $x, y \in A$ or $x, y \in B$ then $[x, y] \subset \mathcal{O}_{\max \left\{\varepsilon_{1}, \varepsilon_{2}\right\}}(C)$. So, assume that $x \in A, y \in B$. Fix $a \in A, b \in B$, and consider the geodesic quadrangle $x y b a$ (see Figure 1).

By remark 0 we have $[x, y] \subset \mathcal{O}_{2 \delta}([x, a] \cup[a, b] \cup[b, y])$. After denoting $d(a, b)=2 \eta$ we obtain $[x, a] \subset \mathcal{O}_{\varepsilon_{1}}(A),[b, y] \subset \mathcal{O}_{\varepsilon_{2}}(B),[a, b] \subset \mathcal{O}_{\eta}(A \cup B)$. Hence $[x, a] \cup[a, b] \cup[b, y] \subset \mathcal{O}_{\max \left\{\varepsilon_{1}, \varepsilon_{2}, \eta\right\}}(C)$, consequently, $[x, y] \subset \mathcal{O}_{\max }\left\{\varepsilon_{1}, \varepsilon_{2}, \eta\right\}+2 \delta(C)$, and the lemma is proved

Proof of Proposition 1. Assume $n=2$ (for $n>2$ the statement will follow by induction).

So, let $A, B$ be $\varepsilon_{i}$-quasiconvex subsets of $G$ respectively, $i=1,2$.

Consider arbitrary $a_{1} b_{1}, a_{2} b_{2} \in A B, a_{i} \in A, b_{i} \in B_{i}, i=1,2$, and fix an element $b \in B,|b|_{G}=\eta$. Then, since the triangles are $\delta$-slim,

$$
\left[b_{1}, 1_{G}\right] \subset \mathcal{O}_{\delta}\left(\left[b, 1_{G}\right] \cup\left[b, b_{1}\right]\right) \subset \mathcal{O}_{\delta+\eta}\left(\left[b, b_{1}\right]\right) \subset \mathcal{O}_{\delta+\eta+\varepsilon_{2}}(B) .
$$

Denoting $\varepsilon_{3}=\delta+\eta+\varepsilon_{2}$, one obtains $\left[b_{1}, 1_{G}\right] \subset \mathcal{O}_{\varepsilon_{3}}(B)$ and, similarly, $\left[b_{2}, 1_{G}\right] \subset \mathcal{O}_{\varepsilon_{3}}(B)$. Therefore, $\left[a_{1} b_{1}, a_{1}\right] \subset \mathcal{O}_{\varepsilon_{3}}\left(a_{1} B\right),\left[a_{2} b_{2}, a_{2}\right] \subset \mathcal{O}_{\varepsilon_{3}}\left(a_{2} B\right)$. Also, observe that $\forall a \in A \quad d(a, a b)=|b|_{G}=\eta$, i.e. $A \subset \mathcal{O}_{\eta}(A b) \subset \mathcal{O}_{\eta}(A B)$, hence $\left[a_{1}, a_{2}\right] \subset \mathcal{O}_{\varepsilon_{1}}(A) \subset \mathcal{O}_{\varepsilon_{1}+\eta}(A B)$. And using remark 0 we achieve

$$
\left[a_{1} b_{1}, a_{2} b_{2}\right] \subset \mathcal{O}_{2 \delta}\left(\left[a_{1} b_{1}, a_{1}\right] \cup\left[a_{1}, a_{2}\right] \cup\left[a_{2} b_{2}, a_{2}\right]\right) \subset \mathcal{O}_{2 \delta+\max \left\{\varepsilon_{1}+\eta, \varepsilon_{3}\right\}}(A B),
$$

q.e.d.

Corollary 1. In a hyperbolic group $G$ every quasiconvex product is a quasiconvex set .

This follows directly from the proposition 1 and part (a) of remark 4 .

\section{Intersections of quasiconvex products}

Set a partial order on $\mathbb{Z}^{2}:\left(a_{1}, b_{1}\right) \leq\left(a_{2}, b_{2}\right)$ if $a_{1} \leq a_{2}$ and $b_{1} \leq b_{2}$. As usual, $\left(a_{1}, b_{1}\right)<\left(a_{2}, b_{2}\right)$ if $\left(a_{1}, b_{1}\right) \leq\left(a_{2}, b_{2}\right)$ and $\left(a_{1}, b_{1}\right) \neq\left(a_{2}, b_{2}\right)$. 
Definition : a finite sequence $\left(\left(i_{1}, j_{1}\right),\left(i_{2}, j_{2}\right), \ldots,\left(i_{t}, j_{t}\right)\right)$ of pairs of positive integers will be called increasing if it is empty $(t=0)$ or (if $t>0$ ) $\left(i_{q}, j_{q}\right)<\left(i_{q+1}, j_{q+1}\right) \forall q=1,2, \ldots, t-1$. This sequence will also be called $(n, m)$-increasing $(n, m \in \mathbb{N})$ if $1 \leq i_{q} \leq n, 1 \leq j_{q} \leq m$ for all $q \in\{1,2, \ldots, t\}$.

Note that the length of an $(n, m)$-increasing sequence never exceeds $(n+m-1)$.

Instead of proving theorem 1 we will prove

Theorem $1^{\prime}$. Suppose $G_{1}, \ldots, G_{n}, H_{1}, \ldots, H_{m}$ are quasiconvex subgroups of the group $G, n, m \in \mathbb{N} ; f, e \in G$. Then there exist numbers $r, t_{l} \in \mathbb{N} \cup\{0\}$ and $f_{l}, \alpha_{l k}, \beta_{l k} \in G, k=1,2, \ldots, t_{l}$ (for every fixed $l$ ), $l=1,2, \ldots, r$, such that

$$
f G_{1} G_{2} \cdot \ldots \cdot G_{n} \cap e H_{1} H_{2} \cdot \ldots \cdot H_{m}=\bigcup_{l=1}^{r} f_{l} S_{l}
$$

where for each $l, t=t_{l}$, there are indices $1 \leq i_{1} \leq i_{2} \leq \ldots \leq i_{t} \leq n, 1 \leq j_{1} \leq$ $\leq j_{2} \leq \ldots \leq j_{t} \leq m$ :

$$
S_{l}=\left(G_{i_{1}}^{\alpha_{l 1}} \cap H_{j_{1}}^{\beta_{l 1}}\right) \cdot \ldots \cdot\left(G_{i_{t}}^{\alpha_{l t}} \cap H_{j_{t}}^{\beta_{l t}}\right),
$$

and the sequence $\left(\left(i_{1}, j_{1}\right), \ldots,\left(i_{t}, j_{t}\right)\right)$ is $(n, m)$-increasing.

For our convenience, let us also introduce the following

Definition : the unions as in the right-hand side of (1) will be called special. $S_{l}$ as in (2) will be called increasing $(n, m)$-products.

Lemma 3.1. Consider a geodesic polygon $X_{0} X_{1} \ldots X_{n}$ in the Cayley graph $\Gamma(G, \mathcal{A}), n \geq 2$. Then there are points $\bar{X}_{i} \in\left[X_{i} ; X_{i+1}\right], i=1,2, \ldots, n-1$, such that setting $\bar{X}_{0}=X_{0}, \bar{X}_{n}=X_{n}$, we have $\left(\bar{X}_{i-1} \mid \bar{X}_{i+1}\right)_{\bar{X}_{i}} \leq \delta$ and $d\left(\bar{X}_{i},\left[\bar{X}_{i-1} ; X_{i}\right]\right) \leq \delta$, for $1 \leq i \leq n-1$.

Proof of the lemma. First, we recursively construct the vertices $\bar{X}_{i}$. Let $\bar{X}_{1} \in\left[X_{1} ; X_{2}\right], \bar{U}_{1} \in\left[X_{0} ; X_{1}\right]$ be the "special" points of the geodesic triangle $X_{0} X_{1} X_{2}$, i.e. $\left|X_{1}-\bar{X}_{1}\right|=\left|X_{1}-\bar{U}_{1}\right|=\left(X_{0} \mid X_{2}\right)_{X_{1}}$. Now, if $\bar{X}_{i-1}$ is constructed, denote by $\bar{X}_{i} \in\left[X_{i} ; X_{i+1}\right], \bar{U}_{i} \in\left[\bar{X}_{i-1} ; X_{i}\right]$ the special points of triangle $\bar{X}_{i-1} X_{i} X_{i+1}\left(\left|X_{i}-\bar{X}_{i}\right|=\left|X_{i}-\bar{U}_{i}\right|=\left(\bar{X}_{i-1} \mid X_{i+1}\right)_{X_{i}}\right)$. (Figure 2)

Then $d\left(\bar{X}_{i},\left[\bar{X}_{i-1} ; X_{i}\right]\right) \leq\left|\bar{X}_{i}-\bar{U}_{i}\right| \leq \delta, \forall i=1,2, \ldots, n-1$.

For the other part of the claim we will use induction on $n$. $n=2$, then

$$
\begin{gathered}
\left(X_{0} \mid X_{2}\right)_{\bar{X}_{1}} \stackrel{\text { def }}{=} \frac{1}{2}\left(\left|X_{0}-\bar{X}_{1}\right|+\left|X_{2}-\bar{X}_{1}\right|-\left|X_{0}-X_{2}\right|\right) \leq \\
\leq \frac{1}{2}\left(\left|X_{0}-\bar{U}_{1}\right|+\left|\bar{U}_{1}-\bar{X}_{1}\right|+\left|X_{2}-\bar{X}_{1}\right|-\left|X_{0}-X_{2}\right|\right)=\frac{1}{2}\left|\bar{U}_{1}-\bar{X}_{1}\right| \leq \frac{\delta}{2} \leq \delta \quad .
\end{gathered}
$$

Suppose, now, that $n \geq 3$. Let us evaluate the Gromov product $\left(\bar{X}_{0} \mid \bar{X}_{2}\right)_{\bar{X}_{1}}$.

$$
\left(\bar{X}_{0} \mid \bar{X}_{2}\right)_{\bar{X}_{1}}=\frac{1}{2}\left(\left|X_{0}-\bar{X}_{1}\right|+\left|\bar{X}_{2}-\bar{X}_{1}\right|-\left|X_{0}-\bar{X}_{2}\right|\right),
$$




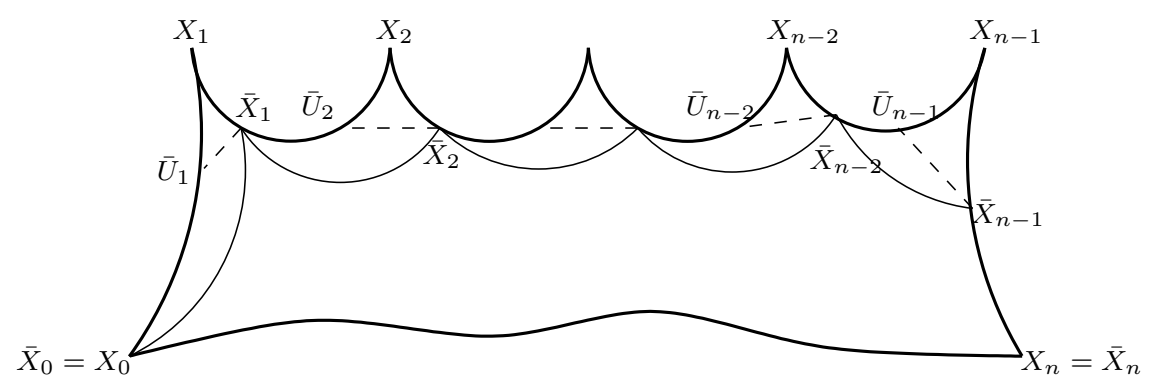

Figure 2

$\left|\bar{X}_{2}-\bar{X}_{1}\right| \leq\left|\bar{X}_{1}-\bar{U}_{2}\right|+\left|\bar{X}_{2}-\bar{U}_{2}\right| \leq\left|\bar{X}_{1}-\bar{U}_{2}\right|+\delta,\left|X_{0}-\bar{X}_{1}\right| \leq\left|X_{0}-\bar{U}_{1}\right|+\delta$, $\left|X_{0}-\bar{U}_{1}\right|+\left|X_{2}-\bar{X}_{1}\right|=\left|X_{0}-X_{2}\right|-$ by the definition of special points of the triangle $X_{0} X_{1} X_{2}$. Therefore

$$
\begin{gathered}
\left|X_{0}-\bar{X}_{1}\right|+\left|\bar{X}_{2}-\bar{X}_{1}\right| \leq\left|X_{0}-\bar{U}_{1}\right|+\left|\bar{X}_{1}-\bar{U}_{2}\right|+2 \delta= \\
=\left|X_{0}-\bar{U}_{1}\right|+\left(\left|X_{2}-\bar{X}_{1}\right|-\left|X_{2}-\bar{U}_{2}\right|\right)+2 \delta=\left|X_{0}-X_{2}\right|-\left|X_{2}-\bar{X}_{2}\right|+2 \delta .
\end{gathered}
$$

Now we notice that $\left|X_{0}-X_{2}\right|-\left|X_{2}-\bar{X}_{2}\right| \leq\left|X_{0}-\bar{X}_{2}\right|$ and obtain:

$$
\left(\bar{X}_{0} \mid \bar{X}_{2}\right)_{\bar{X}_{1}} \leq \frac{1}{2}\left(\left|X_{0}-\bar{X}_{2}\right|+2 \delta-\left|X_{0}-\bar{X}_{2}\right|\right)=\delta .
$$

To the $n$-gon $\bar{X}_{1} X_{2} \ldots X_{n}$ we can apply the induction hypothesis. The lemma is proved.

Proof of theorem $1^{\prime}$. Define $T=f G_{1} G_{2} \cdot \ldots \cdot G_{n} \cap e H_{1} H_{2} \cdot \ldots \cdot H_{m}$.

Fix some finite generating sets in every $G_{i}, H_{j}$ and denote

$$
\begin{aligned}
& K_{1}=\max \left\{1 ;|f|_{G} ; \mid \text { generators of }\left.G_{i}\right|_{G}: i=1,2, \ldots, n\right\}<\infty, \\
& K_{2}=\max \left\{1 ;|e|_{G} ; \mid \text { generators of }\left.H_{j}\right|_{G}: j=1,2, \ldots, m\right\}<\infty .
\end{aligned}
$$

Induction on $(n+m)$.

If $n=0$ or $m=0$, then $\operatorname{card}(T) \leq 1$ and the statement is true.

Let $n \geq 1$ and $m \geq 1, n+m \geq 2$.

Choose an arbitrary $x \in T, x=f g_{1} g_{2} \cdot \ldots \cdot g_{n}=e h_{1} h_{2} \cdot \ldots \cdot h_{m}$ where $g_{i} \in G_{i}, h_{j} \in H_{j}, i=1, \ldots, n, j=1, \ldots, m$.

Consider a pair of (non-geodesic) polygons associated with $x$ in $\Gamma(G, \mathcal{A})$ : $P=X_{0} p_{1} X_{1} p_{2} \ldots p_{n} X_{n} p_{0}$ and $Q=Y_{0} q_{1} Y_{1} q_{2} \ldots q_{m} Y_{m} q_{0}$ with vertices $X_{0}=Y_{0}=1_{G}, X_{i}=f g_{1} \cdot \ldots \cdot g_{i} \in G, Y_{j}=e h_{1} \cdot \ldots \cdot h_{j} \in G, i=1, \ldots, n$, $j=1, \ldots, m$, and edges $p_{0}, p_{1}, \ldots, p_{n}, q_{0}, q_{1}, \ldots, q_{m}$. Such that $p_{1}$, starting at $X_{0}$ and ending at $X_{1}$, is a union of a geodesic path corresponding to $f$ and a $G_{1}$-path corresponding to $g_{1}, p_{i}$ is a $G_{i}$-path labelled by a word representing the element $g_{i}$ in $G$ from $X_{i-1}$ to $X_{i}, i=2, \ldots, n ; p_{0}$ is the geodesic path $\left[X_{n}, X_{0}\right]$ (Figure 3). 


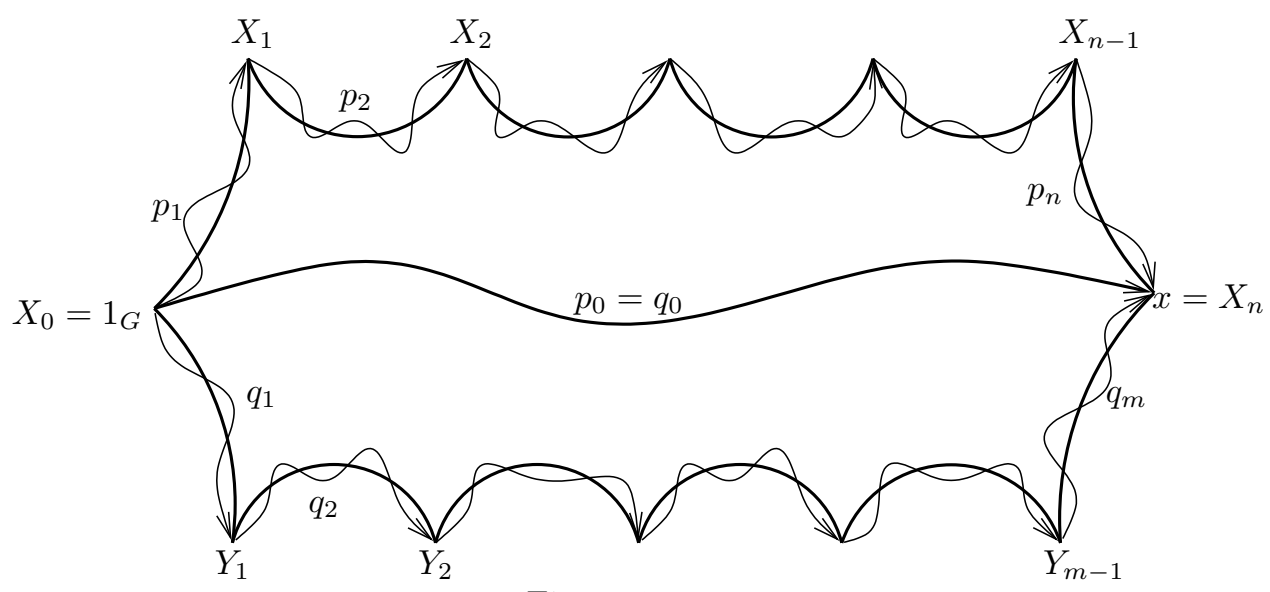

Figure 3

By construction, there are constants $\lambda_{i}, c_{i}$ (not depending on $x \in T$ ) such that the segments $p_{i}, i=1, \ldots, n$ are $\left(\lambda_{i}, c_{i}\right)$-quasigeodesic respectively.

Similarly one constructs the paths $q_{j}, j=0, \ldots, m$.

Therefore the geodesic path $p_{0}=\left[X_{0} ; X_{n}\right]=\left[Y_{0} ; Y_{m}\right]=q_{0}$ will be labelled by a word representing $x$ in our Cayley graph.

We will also consider the geodesic polygons $X_{0} X_{1} \ldots X_{n}$ and $Y_{0} Y_{1} \ldots Y_{m}$ with same vertices as $P$ and $Q$ respectively.

Recalling the property of quasigeodesic paths, for each $i=1, \ldots, n$ $[j=1, \ldots, m]$ we obtain a constant $N_{i}>0\left[M_{j}>0\right]$ (not depending on the element $x \in T$ ) such that

$$
\left[X_{i-1}, X_{i}\right] \subset \mathcal{O}_{N_{i}}\left(p_{i}\right) \quad\left[\left[Y_{j-1}, Y_{j}\right] \subset \mathcal{O}_{M_{j}}\left(q_{j}\right)\right] .
$$

Define $L=\max \left\{N_{1}, \ldots, N_{n}, M_{1}, \ldots, M_{m}\right\}$.

a) Suppose $n, m \geq 2$ (after considering this case, we will see that the other cases, when $n=1$ or $m=1$ are easier) .

Let's focus our attention on the polygons $X_{0} \ldots X_{n}$ and $P$ since everything for the two others can be done analogously.

One can apply lemma 3.1 and obtain $\tilde{X}_{i} \in\left[X_{i} ; X_{i+1}\right], i=1, \ldots, n-1$, such that $\left(\tilde{X}_{i-1} \mid \tilde{X}_{i+1}\right)_{\tilde{X}_{i}} \leq \delta, i=1, \ldots, n-1,\left(\tilde{X}_{0}=X_{0}=1_{G}, \tilde{X}_{n}=X_{n}=x\right)$, along with $\tilde{U}_{i} \in\left[\tilde{X}_{i-1} ; X_{i}\right],\left|\tilde{X}_{i}-\tilde{U}_{i}\right| \leq \delta, i=1, \ldots, n-1$.

Now, using (i), we obtain points $\bar{X}_{i} \in p_{i+1}, i=1, \ldots, n-1$, satisfying $d\left(\tilde{X}_{i}, \bar{X}_{i}\right) \leq L\left(\bar{X}_{0}=X_{0}=1_{G}, \bar{X}_{n}=X_{n}=x\right)$ and $\bar{U}_{1} \in p_{1}$ satisfying $d\left(\tilde{U}_{1}, \bar{U}_{1}\right) \leq L$. For each $i \in\{1,2, \ldots, n-2\}$ the triangle $\tilde{X}_{i} \bar{X}_{i} X_{i+1}$ is $\delta$-slim, hence $\exists \tilde{U}_{i+1}^{\prime} \in\left[\bar{X}_{i}, X_{i+1}\right]: d\left(\tilde{U}_{i+1}^{\prime}, \tilde{U}_{i+1}\right) \leq L+\delta$. The segment of $p_{i+1}$ between $\bar{X}_{i}$ and $X_{i+1}$ is quasigeodesic with the same constants as $p_{i+1}$, therefore there is a point $\bar{U}_{i+1} \in p_{i+1}$ between $\bar{X}_{i}$ and $X_{i+1}$ such that $d\left(\tilde{U}_{i+1}^{\prime}, \bar{U}_{i+1}\right) \leq L$, and, consequently, $d\left(\tilde{U}_{i+1}, \bar{U}_{i+1}\right) \leq 2 L+\delta$ (see Figure 4$)$. 


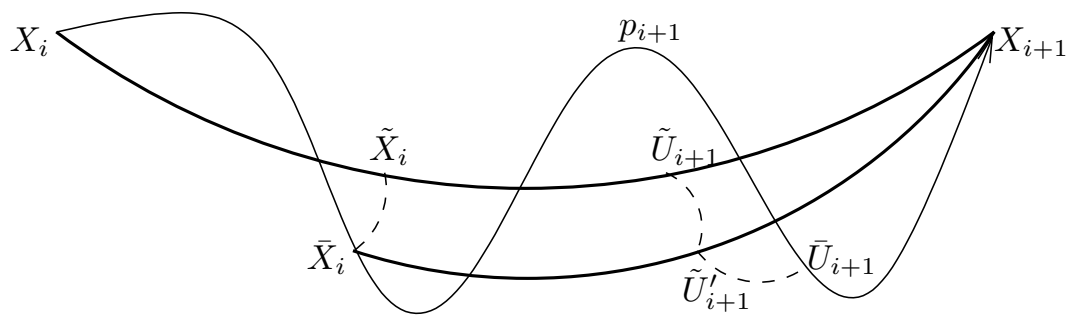

Figure 4

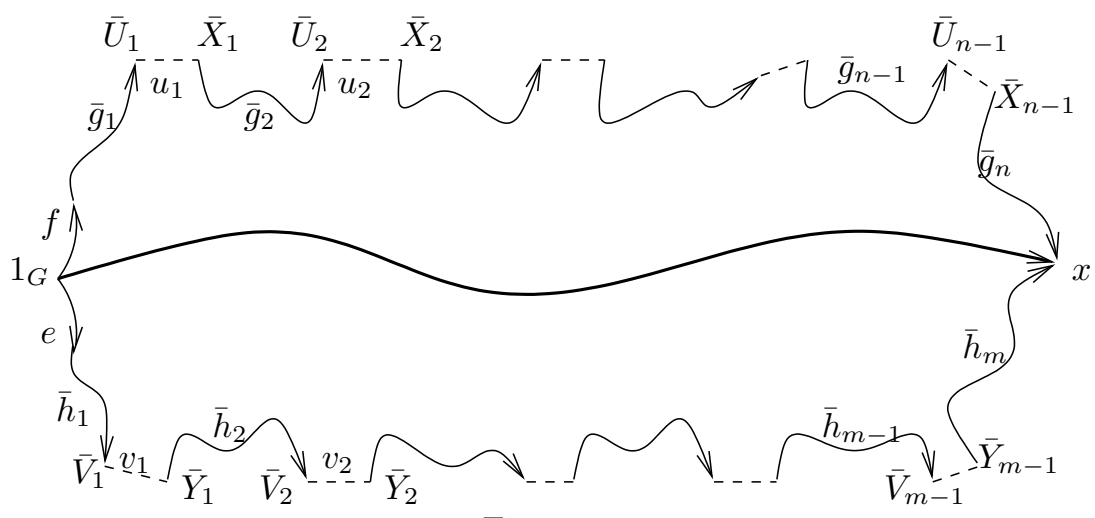

Figure 5

Let $\alpha_{t}$ denote the segment of $p_{t}$ from $\bar{X}_{t-1}$ to $X_{t}, t=2, \ldots, n$, and

$\beta_{s}$ - the subpath of $p_{s}$ from $\bar{X}_{s-1}$ to $\bar{U}_{s}, s=1, \ldots, n-1$. Shifting the points $\bar{X}_{i}, \bar{U}_{i}, i=1, \ldots, n-1$, along their sides of $P$ ( so that $\bar{U}_{i}$ still stays between $\bar{X}_{i-1}$ and $X_{i}$ on $\left.p_{i}\right)$ by distances at most $K_{1}$, we can achieve elem $\left(\beta_{1}\right) \in f G_{1}$ (i.e. $\operatorname{lab}\left(\beta_{1}\right)$ represents an element of $\left.f G_{1}\right), \operatorname{elem}\left(\alpha_{t}\right) \in G_{t+1}, \operatorname{elem}\left(\beta_{s}\right) \in G_{s}$, $t=2, \ldots, n, s=1,2, \ldots, n-1$. And after this, setting, for brevity, $K=\max \left\{K_{1}+\frac{3}{2} L, K_{2}+\frac{3}{2} L\right\}$, one obtains

$$
\begin{gathered}
\left(\bar{X}_{i-1} \mid \bar{X}_{i+1}\right)_{\bar{X}_{i}} \leq \delta+3 K_{1}+3 L \leq \delta+3 K \leq 14 \delta+3 K \stackrel{\text { def }}{=} C_{0}, \\
\left|\bar{X}_{i}-\bar{U}_{i}\right| \leq \delta+2 K_{1}+3 L+\delta \leq 2 \delta+2 K, i=1, \ldots, n-1 .
\end{gathered}
$$

Let $\operatorname{elem}\left(\beta_{1}\right)=f \bar{g}_{1}, \operatorname{elem}\left(\beta_{i}\right)=\bar{g}_{i}, i=1, \ldots, n-1, \operatorname{elem}\left(\alpha_{n}\right)=\bar{g}_{n}$, where $\bar{g}_{k} \in G_{k}, k=1,2, \ldots, n$. elem $\left(\left[\bar{U}_{i} ; \bar{X}_{i}\right]\right)=u_{i} \in G_{i} G_{i+1}, i=1, \ldots, n-1$.

Then $\left|u_{i}\right|_{G} \leq 2 \delta+2 K$, and there are only finitely many of possible $u_{i}$ 's for every $i \in\{1,2, \ldots, n-1\}$. Hence, we achieved the following representation for $x$ :

$$
x \stackrel{G}{=} f \bar{g}_{1} u_{1} \bar{g}_{2} u_{2} \cdot \ldots \cdot \bar{g}_{n-1} u_{n-1} \bar{g}_{n} .
$$

Similarly, one can obtain

$$
x \stackrel{G}{=} e \bar{h}_{1} v_{1} \bar{h}_{2} v_{2} \cdot \ldots \cdot \bar{h}_{m-1} v_{m-1} \bar{h}_{m},
$$


where $\bar{h}_{j} \in H_{j}, j=1, \ldots, m ; v_{j} \in H_{j} H_{j+1}$ and $\left|v_{j}\right|_{G} \leq 2 \delta+2 K$ for every $j=1,2, \ldots, m-1$ (see Figure 5 ).

$\mathcal{U}_{i} \stackrel{\text { def }}{=}\left\{u \in G_{i} G_{i+1}:|u|_{G} \leq 2 \delta+2 K\right\} \subset G, i=1, \ldots, n-1 . \operatorname{card}\left(\mathcal{U}_{i}\right)<\infty$, $\forall i=1, \ldots, n-1$. For convenience, $\mathcal{U}_{0}=\mathcal{U}_{n}=G_{0}=G_{n+1} \stackrel{\text { def }}{=}\left\{1_{G}\right\}$.

Analogously, define $\mathcal{V}_{j} \subset H_{j} H_{j+1}, j=1, \ldots, m-1$, and again, $\mathcal{V}_{0}=\mathcal{V}_{m}=H_{0}=H_{m+1} \stackrel{\text { def }}{=}\left\{1_{G}\right\}$

Set $D=14\left(\delta+C_{0}\right)+3 K=$ const, and $\mathcal{L}=\left\{g \in G:|g|_{G} \leq D\right\}$. At last, we denote

$$
\begin{gathered}
\Delta_{i}=\mathcal{U}_{i-1} \cdot\left(\mathcal{L} \cap G_{i}\right) \cdot \mathcal{U}_{i} \subset G_{i-1} G_{i} G_{i+1} \subset G, i=1,2, \ldots, n, \\
\Theta_{i}=\mathcal{V}_{j-1} \cdot\left(\mathcal{L} \cap H_{j}\right) \cdot \mathcal{V}_{j} \subset H_{j-1} H_{j} H_{j+1} \subset G, j=1,2, \ldots, m .
\end{gathered}
$$

By construction, $\operatorname{card}\left(\Delta_{i}\right)<\infty, \operatorname{card}\left(\Theta_{j}\right)<\infty, \forall i, j$.

Take any $i \in\{1,2, \ldots, n\}$ and consider the intersection

$$
\begin{gathered}
T \supseteq f G_{1} G_{2} \cdot \ldots \cdot G_{i-1} \Delta_{i} G_{i+1} \cdot \ldots \cdot G_{n} \cap e H_{1} \cdot \ldots \cdot H_{m}= \\
=\bigcup_{g \in \Delta_{i}}\left[f G_{1} G_{2} \cdot \ldots \cdot G_{i-1} g G_{i+1} \cdot \ldots \cdot G_{n} \cap e H_{1} \cdot \ldots \cdot H_{m}\right]= \\
=\bigcup_{g \in \Delta_{i}}\left[f g\left(g^{-1} G_{1} g\right)\left(g^{-1} G_{2} g\right) \cdot \ldots\left(g^{-1} G_{i-1} g\right) G_{i+1} \cdot \ldots \cdot G_{n} \cap e H_{1} \cdot \ldots \cdot H_{m}\right] .
\end{gathered}
$$

Because of remark 4, one can apply the induction hypothesis to the last expression and conclude that it is a (finite) "special" union. Hence,

$$
T_{1} \stackrel{\text { def }}{=} \bigcup_{i=1}^{n}\left(f G_{1} G_{2} \cdot \ldots \cdot G_{i-1} \Delta_{i} G_{i+1} \cdot \ldots \cdot G_{n} \cap e H_{1} \cdot \ldots \cdot H_{m}\right)
$$

is also a finite special union.

Because of the symmetry, we parallely showed that

$$
T_{2} \stackrel{\text { def }}{=} \bigcup_{j=1}^{m}\left(f G_{1} \cdot \ldots \cdot G_{n} \cap e H_{1} H_{2} \cdot \ldots \cdot H_{j-1} \Theta_{j} H_{j+1} \cdot \ldots \cdot H_{m}\right)
$$

is a finite "special" union.

We have just proved that there exist $r_{1} \in \mathbb{N} \cup\{0\}, f_{l} \in G$ and increasing $(n, m)$-products $S_{l}, l=1,2, \ldots, r_{1}$, such that

$$
T_{1} \cup T_{2}=\bigcup_{l=1}^{r_{1}} f_{l} S_{l} \subseteq T .
$$

$T=T_{1} \cup T_{2} \cup T_{3}$, where $T_{3} \stackrel{\text { def }}{=} T \backslash\left(T_{1} \cup T_{2}\right)$. Now, let's consider the case $x \in T_{3}$. It means that in representations $(*)$ and $(* *)$ for $x,\left|\bar{g}_{i}\right|_{G}>D$, $\left|\bar{h}_{j}\right|_{G}>D$, for $D=14\left(\delta+C_{0}\right)+3 K$ and $\forall i=1, \ldots, n, \forall j=1, \ldots, m$. Therefore, returning to the pair of polygons we constructed, one will have : 


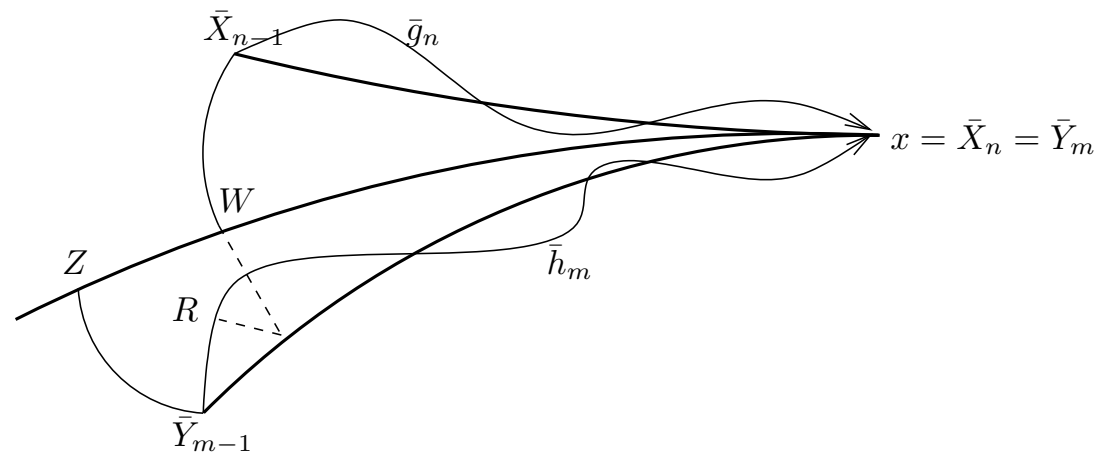

Figure 6

||$\left[\bar{X}_{0} ; \bar{X}_{1}\right] \| \geq\left|\bar{g}_{1}\right|_{G}-|f|_{G}-\left|u_{1}\right|_{G}>14\left(\delta+C_{0}\right)+3 K-K-2 \delta-2 K=$ $=12\left(\delta+C_{0}\right)+2 C_{0} \stackrel{\text { def }}{=} C_{1},\left\|\left[\bar{X}_{i-1} ; \bar{X}_{i}\right]\right\| \geq\left|\bar{g}_{i}\right|_{G}-\left|u_{i}\right|_{G}>14\left(\delta+C_{0}\right)+3 K-2 \delta-$ $-2 K>C_{1}, i=2, \ldots, n-1,\left\|\left[\bar{X}_{n-1} ; \bar{X}_{n}\right]\right\|=\left|\bar{g}_{n}\right|_{G}>C_{1}$.

We also possess the following inequalities : $\left(\bar{X}_{i-1} \mid \bar{X}_{i+1}\right)_{\bar{X}_{i}}<C_{0}, i=1, \ldots, n-1$, $C_{0} \geq 14 \delta, C_{1}>12\left(\delta+C_{0}\right)$.

By lemma 1.3 , the broken line $\left[\bar{X}_{0} ; \bar{X}_{1} ; \ldots ; \bar{X}_{n}\right]$ is contained in the closed $C=2 C_{0}$-neighborhood of the geodesic segment $\left[\bar{X}_{0} ; \bar{X}_{n}\right]$. In particular,

$$
d\left(\bar{X}_{n-1},\left[\bar{X}_{0} ; \bar{X}_{n}\right]\right) \leq C .
$$

A similar argument shows that $d\left(\bar{Y}_{m-1},\left[\bar{Y}_{0} ; \bar{Y}_{m}\right]\right) \leq C$, and, since $\left[\bar{X}_{0} ; \bar{X}_{n}\right]=$ $=\left[X_{0} ; X_{n}\right]=\left[Y_{0} ; Y_{m}\right]=\left[\bar{Y}_{0} ; \bar{Y}_{m}\right]$, one has

$$
d\left(\bar{Y}_{m-1},\left[\bar{X}_{0} ; \bar{X}_{n}\right]\right) \leq C .
$$

b) In the previous case we assumed that $n, m \geq 2$ and we needed quite a long argument to prove (5) and (6). On the other hand, if, for example, $n=1$, then $X_{0}=\bar{X}_{n-1}$ and (5) is trivial.

Because of (5) and (6) one can choose $W, Z \in\left[X_{0} ; X_{n}\right]$ with the properties $\left|W-\bar{X}_{n-1}\right| \leq C,\left|Z-\bar{Y}_{m-1}\right| \leq C$.

The first possibility is, when the point $W$ on $\left[X_{0} ; X_{n}\right]$ lies between $Z$ and $X_{n}$, i.e. $W \in\left[Z ; X_{n}\right]$.

Then, since triangles are $\delta$-thin in the hyperbolic space $\Gamma(G, \mathcal{A})$, $d\left(W,\left[\bar{Y}_{m-1} ; X_{n}\right]\right) \leq C+\delta$. Hence $d\left(\bar{X}_{n-1},\left[\bar{Y}_{m-1} ; X_{n}\right]\right) \leq 2 C+\delta$. Consequently, because $q_{m}$ is quasigeodesic, there exists a point $R$ on the subpath $\gamma$ of $q_{m}$ from $\bar{Y}_{m-1}$ to $Y_{m}$ such that $d\left(\bar{X}_{n-1}, R\right) \leq 2 C+\delta+K+M_{m}\left(M_{m}\right.$ is the same as in (i) ) and $\operatorname{elem}\left(\left[R ; Y_{m}\right]\right)=\operatorname{elem}(\gamma)=\hat{h}_{m} \in H_{m}$.

Define $\Omega=\left\{g \in G_{n} H_{m}:|g|_{G} \leq 2 C+\delta+K+M_{m}\right\}$. Therefore $\operatorname{card}(\Omega)<\infty$ and $\operatorname{elem}\left(\left[\bar{X}_{n-1} ; R\right]\right) \in \Omega$.

For each element $g \in \Omega$ take a pair $g^{\prime} \in G_{n}, h^{\prime} \in H_{m}$ such that $g=g^{\prime} h^{\prime}$. By $G^{\prime} \subset G_{n}$ denote the set of all elements $g^{\prime}$ which we have chosen, by $H^{\prime} \subset H_{m}$ - the set of all $h^{\prime}$ 's.

$$
x=f \bar{g}_{1} u_{1} \ldots u_{n-1} \bar{g}_{n}=e \bar{h}_{1} v_{1} \ldots v_{m-1} \bar{h}_{m} .
$$


From the triangle $\bar{X}_{n-1} X_{n} R$ we obtain $\bar{g}_{n} \hat{h}_{m}^{-1}=g^{\prime} h^{\prime} \in \Omega, g^{\prime} \in G^{\prime}, h^{\prime} \in H^{\prime}$. Thus $\left(g^{\prime}\right)^{-1} \bar{g}_{n}=h^{\prime} \hat{h}_{m} \in G_{n} \cap H_{m}$.

$$
\begin{aligned}
& x \in f G_{1} G_{2} \cdot \ldots \cdot G_{n-1} \cdot u_{n-1} g^{\prime} \cdot\left(\left(g^{\prime}\right)^{-1} \bar{g}_{n}\right) \cap e H_{1} H_{2} \cdot \ldots \cdot H_{m-1} H_{m} \subset \\
& \subset f G_{1} G_{2} \cdot \ldots \cdot G_{n-1} \mathcal{U}_{n-1} G^{\prime} \cdot\left(G_{n} \cap H_{m}\right) \cap e H_{1} H_{2} \cdot \ldots \cdot H_{m-1} H_{m} \subset T .
\end{aligned}
$$

Denote $I=\mathcal{U}_{n-1} \cdot G^{\prime} \subset G_{n-1} G_{n}$ - a finite subset of $G$. Then

$$
\begin{aligned}
x & \in f G_{1} G_{2} \cdot \ldots \cdot G_{n-1} I \cdot\left(G_{n} \cap H_{m}\right) \cap e H_{1} H_{2} \cdot \ldots \cdot H_{m-1} H_{m}= \\
& =\left[f G_{1} G_{2} \cdot \ldots \cdot G_{n-1} I \cap e H_{1} H_{2} \cdot \ldots \cdot H_{m}\right] \cdot\left(G_{n} \cap H_{m}\right) \subset T .
\end{aligned}
$$

The second possibility, when $Z \in\left[W ; X_{n}\right]$ is considered analogously, and, in this case, one obtains a finite subset $J \subset H_{m-1} H_{m}$ such that

$$
x \in\left[f G_{1} G_{2} \cdot \ldots \cdot G_{n} \cap e H_{1} H_{2} \cdot \ldots \cdot H_{m-1} J\right] \cdot\left(G_{n} \cap H_{m}\right) \subset T .
$$

Therefore, we showed that $T_{3} \subseteq\left[T_{3}^{\prime} \cup T_{3}^{\prime \prime}\right] \cdot\left(G_{n} \cap H_{m}\right) \subset T$ where

$$
\begin{aligned}
& T_{3}^{\prime} \stackrel{\text { def }}{=} f G_{1} G_{2} \cdot \ldots \cdot G_{n-1} I \cap e H_{1} H_{2} \cdot \ldots \cdot H_{m}, \\
& T_{3}^{\prime \prime} \stackrel{\text { def }}{=} f G_{1} G_{2} \cdot \ldots \cdot G_{n} \cap e H_{1} H_{2} \cdot \ldots \cdot H_{m-1} J .
\end{aligned}
$$

Combining the formulas (3),(4),(7),(8) and the property that if $H \leq G$ and $a \in H$ then $a H=H a=H$, we obtain the following

Lemma 3.2. In notations of the theorem 1

$$
f G_{1} G_{2} \cdot \ldots \cdot G_{n} \cap e H_{1} H_{2} \cdot \ldots \cdot H_{m}=T_{1} \cup T_{2} \cup\left[T_{3}^{\prime} \cup T_{3}^{\prime \prime}\right] \cdot\left(G_{n} \cap H_{m}\right)
$$

where

$$
\begin{gathered}
T_{1}=\bigcup_{i=1}^{n}\left(f G_{1} G_{2} \cdot \ldots \cdot G_{i-1} \bar{\Delta}_{i} G_{i+1} \cdot \ldots \cdot G_{n} \cap e H_{1} \cdot \ldots \cdot H_{m}\right) \\
T_{2}=\bigcup_{j=1}^{m}\left(f G_{1} \cdot \ldots \cdot G_{n} \cap e H_{1} H_{2} \cdot \ldots \cdot H_{j-1} \bar{\Theta}_{j} H_{j+1} \cdot \ldots \cdot H_{m}\right) \\
T_{3}^{\prime}=f G_{1} G_{2} \cdot \ldots \cdot G_{n-1} \bar{I} \cap e H_{1} H_{2} \cdot \ldots \cdot H_{m} \\
T_{3}^{\prime \prime}=f G_{1} G_{2} \cdot \ldots \cdot G_{n} \cap e H_{1} H_{2} \cdot \ldots \cdot H_{m-1} \bar{J}
\end{gathered}
$$

for some finite subsets $\bar{\Delta}_{i} \subset G_{i}, \bar{\Theta}_{j} \subset H_{j}, \bar{I} \subset G_{n}, \bar{J} \subset H_{m}, 1 \leq i \leq n$, $1 \leq j \leq m$.

Now, to finish the proof of the theorem, we apply the inductive hypothesis:

$$
T_{3} \subseteq \bigcup_{g \in I}\left[f G_{1} G_{2} \cdot \ldots \cdot G_{n-1} g \cap e H_{1} H_{2} \cdot \ldots \cdot H_{m}\right] \cdot\left(G_{n} \cap H_{m}\right) \cup
$$




$$
\begin{gathered}
\cup \bigcup_{h \in J}\left[f G_{1} G_{2} \cdot \ldots \cdot G_{n} \cap e H_{1} H_{2} \cdot \ldots \cdot H_{m-1} h\right] \cdot\left(G_{n} \cap H_{m}\right)= \\
=\bigcup_{g \in I}\left[f g G_{1}^{g^{-1}} G_{2}^{g^{-1}} \cdot \ldots \cdot G_{n-1}^{g^{-1}} \cap e H_{1} H_{2} \cdot \ldots \cdot H_{m}\right] \cdot\left(G_{n} \cap H_{m}\right) \cup \\
\cup \bigcup_{h \in J}\left[f G_{1} G_{2} \cdot \ldots \cdot G_{n} \cap e h H_{1}^{h^{-1}} H_{2}^{h^{-1}} \cdot \ldots \cdot H_{m-1}^{h^{-1}}\right] \cdot\left(G_{n} \cap H_{m}\right)= \\
=\left(\bigcup_{g \in I}\left[\bigcup_{k=1}^{\tilde{r}} \tilde{f}_{k} \tilde{S}_{k}\right] \cup \bigcup_{h \in J}\left[\bigcup_{q=1}^{\hat{r}} \hat{f}_{q} \hat{S}_{q}\right]\right) \cdot\left(G_{n} \cap H_{m}\right)= \\
=\bigcup_{l=r_{1}+1}^{r} f_{l} S_{l} \subset T .
\end{gathered}
$$

Here $\tilde{r}, \hat{r}, r \in \mathbb{N} \cup\{0\}, r \geq r_{1}, \tilde{f}_{k}, \hat{f}_{q}, f_{l} \in G ; \tilde{S}_{k}$ is an (n-1,m)-increasing product, $\hat{S}_{q}$ is an $(\mathrm{n}, \mathrm{m}-1)$-increasing product and $S_{l}$ is an $(\mathrm{n}, \mathrm{m})$-increasing product;

$k=1, \ldots, \tilde{r} ; q=1 \ldots, \hat{r} ; l=r_{1}+1, \ldots, r$.

Hence,

$$
T=T_{1} \cup T_{2} \cup T_{3} \subseteq \bigcup_{l=1}^{r} f_{l} S_{l} \subseteq T
$$

and, thus

$$
T=\bigcup_{l=1}^{r} f_{l} S_{l}
$$

So, the theorem is proved.

Proof of Corollary 2. Observe that arbitrary quasiconvex product $f_{1} G_{1} f_{2} G_{2}$. $\ldots \cdot f_{n} G_{n}$ is equal to a "transformed" product $f G_{1}^{\prime} G_{2}^{\prime} \cdot \ldots \cdot G_{n}^{\prime}$ where $G_{i}^{\prime}=\left(f_{i+1} \cdot \ldots \cdot f_{n}\right)^{-1} G_{i}\left(f_{i+1} \cdot \ldots \cdot f_{n}\right), i=1, \ldots, n-1, G_{n}^{\prime}=G_{n}$, are quasiconvex subgroups of $G$ by remark 4 and $f=f_{1} f_{2} \ldots f_{n} \in G$. It remains to apply theorem 1 to the intersection of "transformed products" several times because a $(n, m)$-increasing product is also a quasiconvex product.

\section{Products of elementary subgroups}

Recall that a group $H$ is called elementary if it has a cyclic subgroup $\langle h\rangle$ of finite index.

Remark 5. An elementary subgroup $H$ of a hyperbolic group $G$ is quasiconvex .

Indeed, we have : $|H:\langle h\rangle|<\infty$. If the element $h$ has a finite order, then $H$ is finite and, thus, quasiconvex. In the case, when the order of $h$ is infinite, by lemmas 1.2,1.1 $\langle h\rangle$ is a quasiconvex subgroup of $G$. By remark 4 and lemma $2.1 \mathrm{H}$ is quasiconvex.

It is well known that any element $x$ of infinite order in $G$ is contained in a unique maximal elementary subgroup $E(x) \leqslant G$ (see [4]). And the intersection 
of two distinct maximal elementary subgroups in a hyperbolic group is finite. Any infinite elementary subgroup contains an element of infinite order.

Obviously, a conjugate subgroup to a maximal elementary subgroup is also maximal elementary.

Proof of Theorem 2. The sufficiency is trivial.

Without loss of generality one can assume $n \geq m$. In this case theorem 2 immediately follows from

Theorem $2^{\prime}$ Let $n \geq m, G_{1}, G_{2}, \ldots, G_{n}, H_{1}, H_{2}, \ldots, H_{m}$ be infinite maximal elementary subgroups of $G, f, e \in G$, and $g_{i} \in G_{i}, i=1,2, \ldots, n$, be elements of infinite order. Also, assume $G_{i} \neq G_{i+1}, H_{j} \neq H_{j+1}, i=1, \ldots, n-1$, $j=1, \ldots, m-1$. If there is a sequence of positive integers $\left(t_{k}\right)_{k=1}^{\infty}$ with the properties:

$$
\lim _{k \rightarrow \infty} t_{k}=\infty \text { and } f g_{1}^{t_{k}} g_{2}^{t_{k}} \cdot \ldots \cdot g_{n}^{t_{k}} \in e H_{1} H_{2} \cdot \ldots \cdot H_{m} \text { for all } k \in \mathbb{N},
$$

then $n=m, G_{n}=H_{n}$, and there exist elements $z_{i} \in H_{i}, i=1, \ldots, n$, such that $G_{i}=\left(z_{n} z_{n-1} \ldots z_{i+1}\right) \cdot H_{i} \cdot\left(z_{n} z_{n-1} \ldots z_{i+1}\right)^{-1}, i=1,2, \ldots, n-1, f=$ $e z_{1}^{-1} z_{2}^{-1} \ldots z_{n}^{-1}$. Consequently, $f G_{1} \cdot \ldots \cdot G_{n}=e H_{1} \cdot \ldots \cdot H_{m}$.

In the conditions of theorem $2^{\prime}$, let $h_{j} \in H_{j}$ be fixed elements of infinite order, $j=1,2, \ldots, m$. Then $G_{i}=E\left(g_{i}\right), H_{j}=E\left(h_{j}\right)$ and $\left|G_{i}:\left\langle g_{i}\right\rangle\right|<\infty$, $\left|H_{j}:\left\langle h_{j}\right\rangle\right|<\infty$. Hence, there exists $T \in \mathbb{N}$ such that for all $j$ and $\forall v \in H_{j}$ $\exists \beta \in \mathbb{Z}, y \in H_{j}: v=y \cdot h_{j}^{\beta}$ and $|y|_{G} \leq T$. Thus, every element $h \in e H_{1} \ldots \cdot H_{m}$ can be presented in the form

$$
h=e y_{1} h_{1}^{\beta_{1}} y_{2} h_{2}^{\beta_{2}} \cdot \ldots \cdot y_{m} h_{m}^{\beta_{m}}
$$

where $\beta_{j} \in \mathbb{Z}, y_{j} \in H_{j},\left|y_{j}\right|_{G} \leq T, j=1,2, \ldots, m$.

Definition: the representation (9) for $h$ will be called reduced if for any $i, j$, $1 \leq i<j \leq m$, such that $\beta_{i}, \beta_{j} \neq 0$, one has

$$
\left(y_{i+1} h_{i+1}^{\beta_{i+1}} \ldots h_{j-1}^{\beta_{j-1}} y_{j}\right)^{-1} \cdot h_{i} \cdot\left(y_{i+1} h_{i+1}^{\beta_{i+1}} \ldots h_{j-1}^{\beta_{j-1}} y_{j}\right) \notin H_{j}=E\left(h_{j}\right) .
$$

Observe that each element $h \in e H_{1} \cdot \ldots \cdot H_{m}$ has a reduced representation. Indeed, if $\left(y_{i+1} h_{i+1}^{\beta_{i+1}} \ldots h_{j-1}^{\beta_{j-1}} y_{j}\right)^{-1} \cdot h_{i} \cdot\left(y_{i+1} h_{i+1}^{\beta_{i+1}} \ldots h_{j-1}^{\beta_{j-1}} y_{j}\right) \in H_{j}$ for some $1 \leq i<j \leq m$ then there are $\beta_{j}^{\prime} \in \mathbb{Z}, y_{j}^{\prime} \in H_{j},\left|y_{j}^{\prime}\right|_{G} \leq T$ :

$$
y_{j} \cdot\left(y_{i+1} h_{i+1}^{\beta_{i+1}} \ldots h_{j-1}^{\beta_{j-1}} y_{j}\right)^{-1} \cdot h_{i}^{\beta_{i}} \cdot\left(y_{i+1} h_{i+1}^{\beta_{i+1}} \ldots h_{j-1}^{\beta_{j-1}} y_{j}\right) \cdot h_{j}^{\beta_{j}}=y_{j}^{\prime} h_{j}^{\beta_{j}^{\prime}} .
$$

Therefore,

$$
h=e y_{1} h_{1}^{\beta_{1}} \cdot \ldots \cdot y_{i-1} h_{i-1}^{\beta_{i-1}} y_{i} y_{i+1} h_{i+1}^{\beta_{i+1}} \cdot \ldots \cdot y_{j-1} h_{j-1}^{\beta_{j-1}} y_{j}^{\prime} h_{j}^{\beta_{j}^{\prime}} y_{j+1} h_{j+1}^{\beta_{j+1}} \cdot \ldots \cdot y_{m} h_{m}^{\beta_{m}}
$$

and the number of non-zero $\beta_{k}$ 's is decreased. Continuing this process, we will obtain a reduced representation for $h$ after a finite number of steps . 
Proof of Theorem $2^{\prime}$. Let $h_{j} \in H_{j}, 1 \leq j \leq m, T$, be as above. Induction on $n$.

If $\mathrm{n}=1$, then, evidently, $m=1$, and $\forall k \in \mathbb{N}$ there is $y_{t_{k}} \in H_{1},\left|y_{t_{k}}\right|_{G} \leq T$, and $\beta_{t_{k}} \in \mathbb{Z}$ such that $f g_{1}^{t_{k}}=e y_{t_{k}} h_{1}^{\beta_{t_{k}}}$. Because of having $\lim _{k \rightarrow \infty} t_{k}=\infty$, one can choose $p, q \in \mathbb{N}$ so that $t_{p}<t_{q}$ and $y_{t_{p}}=y_{t_{q}}$. Therefore,

$$
f g_{1}^{t_{p}} h_{1}^{-\beta_{t_{p}}}=e y_{t_{p}}=f g_{1}^{t_{q}} h_{1}^{-\beta_{t_{q}}}
$$

and, thus, $g_{1}^{t_{p}-t_{q}}=h_{1}^{\beta_{t_{p}}-\beta_{t_{q}}}-$ an element of infinite order in the intersection of $G_{1}$ and $H_{1}$. Consequently, $G_{1}=H_{1}$, because these subgroups are maximal elementary.

Assume, now, that $n>1$. For every $k \in \mathbb{N}$ one has

$$
f g_{1}^{t_{k}} g_{2}^{t_{k}} \cdot \ldots \cdot g_{n}^{t_{k}}=e y_{t_{k}} h_{1}^{\beta_{t_{k} 1}} y_{t_{k}} h_{2}^{\beta_{t_{k} 2}} \cdot \ldots \cdot y_{t_{k} m} h_{m}^{\beta_{t_{k} m}}
$$

where the product in the right-hand side is reduced. Obviously, there exists a subsequence $\left(l_{k}\right)_{k=1}^{\infty}$ of $\left(t_{k}\right)$ and $C \in \mathbb{N}$ such that for each $j \in\{1,2, \ldots, m\}$ either $\left|\beta_{l_{k} j}\right| \leq C$ for all $k$ or $\lim _{k \rightarrow \infty}\left|\beta_{l_{k} j}\right|=\infty$.

Therefore, since $\left|y_{l_{k} j}\right|_{G} \leq T \forall k \in \mathbb{N}, \forall j$, there is a subsequence $\left(s_{k}\right)_{k=1}^{\infty}$ of $\left(l_{k}\right)$ such that $y_{s_{k} j}=y_{j} \in H_{j} \forall j$, and if for $j \in\{1, \ldots, m\}$ we had $\left|\beta_{l_{k} j}\right| \leq C$ $\forall k \in \mathbb{N}$ then $\left|\beta_{s_{k} j}\right|=\beta_{j} \in \mathbb{Z} \forall k \in \mathbb{N}$, and $\lim _{k \rightarrow \infty}\left|\beta_{s_{k} j}\right|=\infty$ for all other $j$ 's.

Thus, $\{1,2, \ldots, m\}=J_{1} \cup J_{2}$ where if $j \in J_{1}$ then $\left|\beta_{s_{k} j}\right|=\beta_{j}$ for every $k$, and if $j \in J_{2}$ then $\lim _{k \rightarrow \infty}\left|\beta_{s_{k}, j}\right|=\infty$. Let $J_{2}=\left\{j_{1}, j_{2}, \ldots, j_{\varkappa}\right\} \subset\{1,2, \ldots, m\}$, $j_{1}<j_{2}<\ldots<j_{\varkappa}$, and denote

$$
\begin{gathered}
w_{1}=y_{1}^{-1} \in H_{1} \text { if } j_{1}=1, \text { otherwise, if } j_{1}>1, \\
w_{1}=y_{j_{1}}^{-1} h_{j_{1}-1}^{-\beta_{j_{1}-1}} y_{j_{1}-1}^{-1} \cdot \ldots \cdot h_{1}^{-\beta_{1}} y_{1}^{-1} \in H_{j_{1}} H_{j_{1}-1} \cdot \ldots \cdot H_{1} ; \\
\ldots \ldots \\
w_{\varkappa}=y_{j_{\varkappa}}^{-1} \in H_{j_{\varkappa}} \text { if } j_{\varkappa}=j_{\varkappa-1}+1, \text { otherwise, if } j_{\varkappa}>j_{\varkappa-1}+1, \\
w_{\varkappa}=y_{j_{\varkappa}}^{-1} h_{j_{\varkappa}-1}^{-\beta_{j_{\varkappa}-1}} y_{j_{\varkappa}-1}^{-1} \ldots \cdot h_{j_{\varkappa-1}+1}^{-\beta_{j_{\varkappa-1}+1}} y_{j_{\varkappa-1}+1}^{-1} \in H_{j_{\varkappa}} H_{j_{\varkappa-1}} \cdot \ldots \cdot H_{j_{\varkappa-1}+1} ; \\
w_{\varkappa+1}=1_{G} \text { if } j_{\varkappa}=m, \text { otherwise, if } j_{\varkappa}<m, \\
w_{\varkappa+1}=h_{m}^{-\beta_{m}} y_{m}^{-1} \cdot \ldots \cdot h_{j_{\varkappa}+1}^{-\beta_{j+1}} y_{j_{\varkappa}+1}^{-1} \in H_{m} H_{m-1} \cdot \ldots \cdot H_{j_{\varkappa}+1} .
\end{gathered}
$$

To simplify the formulas, denote $\delta_{k \nu}=-\beta_{s_{k}, j_{\nu}}, 1 \leq \nu \leq \varkappa$. Then $\lim _{k \rightarrow \infty}\left|\delta_{k \nu}\right|=\infty$ for every $\nu=1,2, \ldots, \varkappa$. (10) is equivalent to

$$
u_{k} \stackrel{\text { def }}{=} f g_{1}^{s_{k}} g_{2}^{s_{k}} \cdot \ldots \cdot g_{n-1}^{s_{k}} w_{\varkappa+1} h_{j_{\varkappa}}^{\delta_{k \varkappa}} w_{\varkappa} h_{j_{\varkappa-1}}^{\delta_{k \varkappa-1}} \cdot \ldots \cdot w_{2} h_{j_{1}}^{\delta_{k 1}} w_{1} e^{-1}=1_{G}
$$

So, $\left|u_{k}\right|_{G}=0$ for all $k \in \mathbb{N}$. Denote $K=\max \left\{|f|_{G},\left|w_{1} e^{-1}\right|_{G},\left|w_{2}\right|_{G}\right.$, $\left.\ldots,\left|w_{\varkappa+1}\right|_{G}\right\}$, and assume that $g_{n} \notin w_{\varkappa+1} E\left(h_{j_{\varkappa}}\right) w_{\varkappa+1}^{-1}$. The product in the right-hand side of (10) was reduced, therefore $h_{j_{\nu}} \notin w_{\nu} E\left(h_{j_{\nu-1}}\right) w_{\nu-1}^{-1}, \nu=$ $2,3, \ldots, \varkappa$. Thus, we can apply Lemma 1.8 to (11) and obtain $\lambda>0, c \geq 0$ 
and $M>0$ (depending on $K, g_{1}, \ldots, g_{n}, h_{j_{1}}, \ldots, h_{j_{\varkappa}}$ ) such that if $s_{k} \geq M$ and $\left|\delta_{k \nu}\right| \geq M, \nu=2,3, \ldots, \varkappa$, then $\left|u_{k}\right|_{G} \geq \lambda \cdot s_{k}-c$. Now, by the choice of the sequence $\left(s_{k}\right)$, there exists $N \in \mathbb{N}: s_{k}>M$ and $\left|\delta_{k \nu}\right|>M \forall k \geq N$, $\nu=2,3, \ldots, \varkappa$. Thus, taking $k \geq \max \{N, c / \lambda\}+1$, we achieve a contradiction: $0=\left|u_{k}\right|_{G}<\lambda \cdot s_{k}-c$.

Hence, $g_{n} \in w_{\varkappa+1} E\left(h_{j_{\varkappa}}\right) w_{\varkappa+1}^{-1}$ which implies

$$
G_{n}=E\left(g_{n}\right)=w_{\varkappa+1} E\left(h_{j_{\varkappa}}\right) w_{\varkappa+1}^{-1}=E\left(w_{\varkappa+1} h_{j_{\varkappa}} w_{\varkappa+1}^{-1}\right) .
$$

Consequently, for every $k \in \mathbb{N} \quad w_{\varkappa+1}^{-1} g_{n}^{s_{k}} w_{\varkappa+1} h_{j_{\varkappa}, \delta_{\varkappa}}^{\delta_{\varkappa}}=y_{k j_{\varkappa}}^{\prime} h_{j_{\varkappa}}^{\gamma_{k}} \in H_{j_{\varkappa}}$ where $\left|y_{k j_{\varkappa}}^{\prime}\right|_{G} \leq T$. By passing to a subsequence of $\left(s_{k}\right)$ we can assume that $y_{k j_{\varkappa}}^{\prime}=y_{j_{\varkappa}}^{\prime} \in H_{j_{\varkappa}}$ for every $k$. Therefore

$$
u_{k}=f g_{1}^{s_{k}} g_{2}^{s_{k}} \cdot \ldots \cdot g_{n-1}^{s_{k}} w_{\varkappa+1} y_{j_{\varkappa}}^{\prime} h_{j_{\varkappa}}^{\gamma_{k}} w_{\varkappa} h_{j_{\varkappa-1}}^{\delta_{k,-1}} \cdot \ldots \cdot w_{2} h_{j_{1}}^{\delta_{k 1}} w_{1} e^{-1}=1_{G} \cdot
$$

Suppose $\limsup _{k \rightarrow \infty}\left|\gamma_{k}\right|=\infty$. Since $E\left(g_{n-1}\right)=G_{n-1} \neq G_{n}=E\left(g_{n}\right)$, we have $g_{n-1} \notin w_{\varkappa+1} E\left(h_{j_{\varkappa}}\right) w_{\varkappa+1}^{-1}=w_{\varkappa+1} y_{k j_{\varkappa}}^{\prime} E\left(h_{j_{\varkappa}}\right)\left(y_{k j_{\varkappa}}^{\prime}\right)^{-1} w_{\varkappa+1}^{-1}$ ( because $\left.y_{k j_{\varkappa}}^{\prime} \in H_{j_{\varkappa}}=E\left(h_{j_{\varkappa}}\right)\right)$.

Then for $K^{\prime}=\max \left\{K,\left|w_{\varkappa+1} y_{j_{\varkappa}}^{\prime}\right|_{G}\right\}$ by Lemma 1.8 there exist $\lambda>0, c \geq 0$ and $M>0$ (depending on $K^{\prime}, g_{1}, \ldots, g_{n-1}, h_{j_{1}}, \ldots, h_{j_{\varkappa}}$ ) such that if $s_{k} \geq M$, $\left|\delta_{k \nu}\right| \geq M, \nu=2,3, \ldots, \varkappa$, and $\left|\gamma_{k}\right| \geq M$ then $\left|u_{k}\right|_{G} \geq \lambda \cdot s_{k}-c$. Now, by the assumption on $\left(s_{k}\right)$ and $\left(\gamma_{k}\right)$, there exists $N \in \mathbb{N}, N>c / \lambda$, such that $s_{N}>M$, $\left|\delta_{N \nu}\right|>M, \nu=2,3, \ldots, \varkappa$, and $\left|\gamma_{N}\right|>M$. Which leads us to a contradiction: $0=\left|u_{k}\right|_{G}<\lambda \cdot s_{k}-c$.

Thus, $\left|\gamma_{k}\right| \leq C_{1}$ for some constant $C_{1}$, so, by passing to a subsequence as above, we can assume that $\gamma_{k}=\gamma \forall k \in \mathbb{N}$. Hence, after setting $z_{\varkappa}=w_{\varkappa+1} y_{j_{\varkappa}}^{\prime} h_{j_{\varkappa}}^{\gamma} w_{\varkappa}$, for every natural index $k$ we will have

$$
u_{k}=f g_{1}^{s_{k}} g_{2}^{s_{k}} \cdot \ldots \cdot g_{n-1}^{s_{k}} z_{\varkappa} h_{j_{\varkappa-1}}^{\delta_{k, \varkappa-1}} w_{j_{\varkappa-1}} \cdot \ldots \cdot w_{2} h_{j_{1}}^{\delta_{k 1}} w_{1} e^{-1}=1_{G} .
$$

Which implies $f g_{1}^{s_{k}} g_{2}^{s_{k}} \cdot \ldots \cdot g_{n-1}^{s_{k}} \in e w_{1}^{-1} H_{j_{1}} w_{2}^{-1} H_{j_{2}} \cdot \ldots \cdot w_{j_{\varkappa-1}}^{-1} H_{j_{\varkappa-1}} z_{\varkappa}^{-1}=$ $=u H_{1}^{v_{2}} H_{2}^{v_{3}} \cdot \ldots \cdot H_{j_{\varkappa-1}}^{v_{\varkappa}}$ where $v_{\nu}=z_{\varkappa} w_{\varkappa-1} \cdot \ldots \cdot w_{\nu+1} w_{\nu}, \nu=2,3, \ldots, \varkappa-1$, $v_{\varkappa}=z_{\varkappa}, u=e w_{1}^{-1} w_{2}^{-1} \cdot \ldots \cdot w_{\varkappa-1}^{-1} z_{\varkappa}^{-1}$.

$n-1 \geq m-1 \geq \varkappa-1$ and the other conditions of the theorem $2^{\prime}$ are satisfied, therefore one can apply the induction hypothesis and obtain that $n-1=\varkappa-1$, hence, $\varkappa=m=n, j_{\nu}=\nu, 1 \leq \nu \leq \varkappa$, and, by definition, $w_{\nu}=y_{j_{\nu}}^{-1} \in H_{\nu}$, $\nu=1,2, \ldots, n, w_{\varkappa+1}=1_{G}, z_{\varkappa}=z_{n} \in H_{n}$. And also $G_{n-1}=H_{j_{\varkappa-1}}^{v_{\varkappa}}=H_{n-1}^{z_{n}}$, and there exist $\hat{z}_{i} \in H_{i}, 1 \leq i \leq n-1$, such that

$$
\begin{gathered}
G_{i}=\left(\hat{z}_{n-1}^{v_{n}} \hat{z}_{n-2}^{v_{n-1}} \ldots \hat{z}_{i+1}^{v_{i+2}}\right) \cdot H_{i}^{v_{i+1}} \cdot\left(\hat{z}_{n-1}^{v_{n}} \hat{z}_{n-2}^{v_{n-1}} \ldots \hat{z}_{i+1}^{v_{i+2}}\right)^{-1}= \\
=\left(z_{n} z_{n-1} \cdot \ldots \cdot z_{i+1}\right) \cdot H_{i} \cdot\left(z_{n} z_{n-1} \cdot \ldots \cdot z_{i+1}\right)^{-1}, i=1,2, \ldots, n-2,
\end{gathered}
$$

where $z_{p}=\hat{z}_{p} w_{p} \in H_{p}, 1 \leq p \leq n-1, f=u\left(\hat{z}_{1}^{v_{2}}\right)^{-1}\left(\hat{z}_{2}^{v_{3}}\right)^{-1} \cdot \ldots \cdot\left(\hat{z}_{n-1}^{v_{2}}\right)^{-1}=$ $=e z_{1}^{-1} z_{2}^{-1} \cdot \ldots \cdot z_{n}^{-1}$.

By (12) $G_{n}=E\left(h_{n}\right)=H_{n}$. The proof of the theorem $2^{\prime}$ is finished. 
Suppose $G_{1}, G_{2}, \ldots, G_{n}$ are infinite maximal elementary subgroups of $G$, $f_{1}, \ldots, f_{n} \in G, n \in \mathbb{N} \cup\{0\}$.

Definition : the set $P=f_{1} G_{1} f_{2} G_{2} \cdot \ldots \cdot f_{n} G_{n}$ will be called ME-product. Thus, if $n=0$, we have the empty set. For convenience, we will also consider every element $g \in G$ to be a ME-product. As in the proof of corollary 2, every such ME-product can be brought to a form (however, not unique)

$$
P^{\prime}=f G_{1}^{\prime} G_{2}^{\prime} \cdot \ldots G_{k}^{\prime}
$$

where $0 \leq k \leq n, f \in G, G_{i}^{\prime}$ are infinite maximal elementary subgroups, $i=1,2, \ldots, k$, and $G_{i}^{\prime} \neq G_{i+1}^{\prime}, 1 \leq i \leq k-1$. The number $k$ in this case will be called $\operatorname{rank}$ of the ME-product $P$ (thus, $\operatorname{rank}(P)=\operatorname{rank}\left(P^{\prime}\right)=k \leq n$ ).

A set $U$ which can be presented as a finite union of ME-products has rank $k$, by definition, if $U=\bigcup_{i=1}^{t} P_{i}$, where $P_{i}, i=1, \ldots, t$, are ME-products, and $k=\max \left\{\operatorname{rank}\left(P_{i}\right) \mid 1 \leq i \leq t\right\}$.

Note: an empty set is defined to have rank $(-1)$; any element of the group $G$ is a ME-product of rank 0; thus any finite non-empty subset of $G$ is a finite union of ME-products of rank 0 .

Remark 6. the rank of a ME-product is defined correctly by theorem 2. By theorem $2^{\prime}$ the definition of the rank of a finite union of ME-products is correct.

Lemma 4.1. Suppose $P, R$ are ME-products in a hyperbolic group $G$. Then the intersection $T \stackrel{\text { def }}{=} P \cap R$ is a finite union of ME-products and its rank is at most $\operatorname{rank}(P)$. If $\operatorname{rank}(T)=\operatorname{rank}(P)$ then $T=P$.

Proof. Since a conjugate to an infinite maximal elementary subgroup is also infinite maximal elementary, it follows from theorem 1 that $T$ is a finite union of ME-products $P_{i}, 1 \leq i \leq t$ (for some $t \in \mathbb{N} \cup\{0\}$ ):

$$
T=P \cap R=\bigcup_{i=1}^{t} P_{i} .
$$

For each $i=1, \ldots, t, P_{i} \subseteq P$, therefore by theorem $2^{\prime}, \operatorname{rank}\left(P_{i}\right) \leq \operatorname{rank}(P)$ (otherwise we would get a contradiction), and $\operatorname{rank}\left(P_{i}\right)=\operatorname{rank}(P)$ if and only if $P_{i}=P$. Thus $\operatorname{rank}(T)=\max \left\{\operatorname{rank}\left(P_{i}\right) \mid 1 \leq i \leq t\right\} \leq \operatorname{rank}(P)$. If $\operatorname{rank}(T)=\operatorname{rank}(P)$ then $\operatorname{rank}\left(P_{i}\right)=\operatorname{rank}(P)$ for some $i$, and so, $P_{i}=P=T$. Q.e.d.

As an immediate consequence of lemma 4.1 one obtains

Corollary 3. let $P$ be a ME-product of rank $n$ and $U$ be a finite union of ME-products. Then the set $P \cap U$ is a finite union of ME-products, $\operatorname{rank}(P \cap U) \leq n$, and if $\operatorname{rank}(P \cap U)=n$ then $P \cap U=P$.

Corollary 4. A non-elementary hyperbolic group $G$ can not be equal to a finite union of its ME-products.

Proof. Suppose, by the contrary, that $G$ is a finite union of ME-products: $G=P_{1} \cup \ldots \cup P_{l}$ and $\operatorname{rank}(G)=m$. Since $G$ is not elementary, there exist 
two elements $x, y \in G$ of infinite order such that $E(x) \neq E(y)$. Hence, one can construct a ME-product $P=G_{1} G_{2} \cdot \ldots \cdot G_{m+1}$ in $G$ where $G_{i}=E(x)$ if $i$ is even, and $G_{i}=E(y)$ if $i$ is odd. Consequently, $\operatorname{rank}(P)=m+1$, but $P \subset G$, thus

$$
P \cap G=P=\bigcup_{j=1}^{l}\left(P_{j} \cap P\right) .
$$

By lemma 4.1, $\operatorname{rank}\left(P_{j} \cap P\right) \leq \operatorname{rank}\left(P_{j}\right) \leq m$ for every $j=1,2, \ldots, l$. Therefore, we achieve a contradiction with the definition of rank : $m+1=$ $=\operatorname{rank}(P)=\operatorname{rank}(P \cap G) \leq m$.

A group $H$ is called bounded-generated if it is a product of finitely many cyclic subgroups, i.e. there are elements $x_{1}, x_{2}, \ldots, x_{k} \in H$ such that every $h \in H$ is equal to $x_{1}^{s_{1}} x_{2}^{s_{2}} \cdot \ldots \cdot x_{k}^{s_{k}}$ for some $s_{1}, \ldots, s_{k} \in \mathbb{Z}$.

Corollary 5. Any bounded-generated hyperbolic group is elementary.

Proof. Indeed, any cyclic subgroup of a hyperbolic group either is finite or is contained in some infinite maximal elementary subgroup. Hence, their product is contained in a finite union of ME-products and we can apply corollary 4.

Proof of Theorem 3. Since there exist at most countably many different ME-products in $G$, it is enough to consider only their countable intersections. Let $P_{j i}, 1 \leq i \leq k_{j}, k_{j}, j \in \mathbb{N}$, be ME-products, and $U_{j}=\bigcup_{i=1}^{k_{j}} P_{j i}$ - their finite unions. Let

$$
T=\bigcap_{j=1}^{\infty} U_{j}
$$

One has to show that there exist ME-products $R_{1}, \ldots, R_{s}, s \in \mathbb{N} \cup\{0\}$, such that $T=R_{1} \cup \ldots \cup R_{s}$.

Induct on $n=\operatorname{rank}\left(U_{1}\right)$.

$$
T=\left(\bigcup_{i=1}^{k_{1}} P_{1 i}\right) \cap \bigcap_{j=2}^{\infty} U_{j}=\bigcup_{i=1}^{k_{1}}\left(P_{1 i} \cap \bigcap_{j=2}^{\infty} U_{j}\right)
$$

So, it is enough to consider the case when $k_{1}=1, U_{1}=P_{11}=P$. If $n=0$ then $P$ is finite and there is nothing to prove.

Assume that $n>0$ and let $J \in \mathbb{N}$ be the smallest index such that $P \cap U_{J} \neq P$ (if there is no such $J$ then $T=P$ and the theorem is true). Therefore

$$
T=P \cap \bigcap_{j=J}^{\infty} U_{j}=\left(P \cap U_{J}\right) \cap \bigcap_{j=J+1}^{\infty} U_{j} .
$$

By corollary $3, P \cap U_{J}$ is a finite union of ME-products :

$$
P \cap U_{J}=\bigcup_{l=1}^{t} R_{l}^{\prime}, t \in \mathbb{N} \cup\{0\}
$$


and $\operatorname{rank}\left(P \cap U_{J}\right)<n$ because of the choice of $J$, therefore $\operatorname{rank}\left(R_{l}^{\prime}\right)<n$, $\forall l=1,2, \ldots, t$.

Hence, by the induction hypothesis,

$$
T=\bigcup_{l=1}^{t}\left[R_{l}^{\prime} \cap \bigcap_{j=J+1}^{\infty} U_{j}\right]=\bigcup_{l=1}^{t}\left[R_{l 1} \cup \ldots \cup R_{l s_{l}}\right]
$$

for some ME-products $R_{l 1}, \ldots, R_{l s_{l}}, s_{l} \in \mathbb{N} \cup\{0\}, 1 \leq l \leq t$.

The statement of the theorem 3 fails to be true if maximal elementary subgroups in the definition of ME-products one substitutes by arbitrary elementary subgroups. Below we construct an example to demonstrate that .

Let $G=F(x, y)$ be the free group with two generators, $q_{1}<q_{2}<q_{3}<\ldots$ be an infinite sequence of prime numbers . Define $d_{i}=q_{1} q_{2} \cdot \ldots \cdot q_{i}$,

$c_{i}=q_{1} q_{2} \cdot \ldots \cdot q_{i-1} q_{i}^{2}=d_{i} \cdot q_{i}, i \in \mathbb{N}$, and the sets $P_{i}, i \in \mathbb{N}$, as follows :

$P_{1}=\left\langle x^{d_{1}}\right\rangle$ - cyclic subgroup of $G$ generated by $x^{d_{1}}=x^{q_{1}}$,

$P_{2}=\langle y\rangle \cdot\left\langle y x^{c_{1}} y^{-1}\right\rangle \cdot\left\langle y^{2} x^{d_{2}} y^{-2}\right\rangle \cdot\langle y\rangle$,

$P_{3}=\langle y\rangle \cdot\left\langle y x^{c_{1}} y^{-1}\right\rangle \cdot\left\langle y^{2} x^{c_{2}} y^{-2}\right\rangle \cdot\left\langle y^{3} x^{d_{3}} y^{-3}\right\rangle \cdot\langle y\rangle$,

$P_{i}=\langle y\rangle \cdot\left\langle y x^{c_{1}} y^{-1}\right\rangle \cdot\left\langle y^{2} x^{c_{2}} y^{-2}\right\rangle \cdot \ldots \cdot\left\langle y^{i-1} x^{c_{i-1}} y^{-(i-1)}\right\rangle\left\langle y^{i} x^{d_{i}} y^{-i}\right\rangle \cdot\langle y\rangle$, …...

Now consider the intersection $T=\bigcap_{i=1}^{\infty} P_{i}$. Let us observe that $P_{1} \cap P_{2}=\left\langle x^{c_{1}}\right\rangle \cup\left\langle x^{d_{2}}\right\rangle, \ldots, \bigcap_{i=1}^{k} P_{i}=\left\langle x^{c_{1}}\right\rangle \cup \ldots \cup\left\langle x^{c_{k-1}}\right\rangle \cup\left\langle x^{d_{k}}\right\rangle, \ldots$. Indeed, $P_{1} \cap P_{2}=\left\langle x^{d_{1}}\right\rangle \cap\left(\left\langle x^{c_{1}}\right\rangle \cup\left\langle x^{d_{2}}\right\rangle\right)=\left\langle x^{c_{1}}\right\rangle \cup\left\langle x^{d_{2}}\right\rangle$. Inducting on $k$, we get

$$
\begin{gathered}
\bigcap_{i=1}^{k} P_{i}=\left(\bigcap_{i=1}^{k-1} P_{i}\right) \cap P_{k}=\left\langle x^{c_{1}}\right\rangle \cup \ldots \cup\left\langle x^{c_{k-2}}\right\rangle \cup\left\langle x^{d_{k-1}}\right\rangle \cap\left(\left\langle x^{c_{1}}\right\rangle \cup \ldots \cup\left\langle x^{c_{k-1}}\right\rangle \cup\right. \\
\left.\cup\left\langle x^{d_{k}}\right\rangle\right)=\left\langle x^{c_{1}}\right\rangle \cup \ldots \cup\left\langle x^{c_{k-2}}\right\rangle \cup\left\langle x^{d_{k-1}}\right\rangle \cap\left(\left\langle x^{c_{k-1}}\right\rangle \cup\left\langle x^{d_{k}}\right\rangle\right)= \\
=\left\langle x^{c_{1}}\right\rangle \cup \ldots \cup\left\langle x^{c_{k-1}}\right\rangle \cup\left\langle x^{d_{k}}\right\rangle .
\end{gathered}
$$

Since $\bigcap_{i=1}^{\infty}\left\langle x^{d_{i}}\right\rangle=\{1\}$, therefore $T=\bigcup_{i=1}^{\infty}\left\langle x^{c_{i}}\right\rangle$.

If $q_{1}=2, q_{2}=3, q_{3}=5, \ldots$, is chosen to be the enumeration of all primes, one can show directly that the set $T$ can not be presented as a finite union of products $f_{1} G_{1} f_{2} G_{2} \cdot \ldots f_{n} G_{n}$, where $f_{1}, \ldots, f_{n} \in G$ and $G_{1}, \ldots, G_{n}$ are elementary (in this case cyclic) subgroups of $G$. We are not going to do that, instead we will use a set-theoretical argument : there are only countably many such finite unions, hence there is an infinite sequence of primes $q_{1}<q_{2}<q_{3}<\ldots$ such that the corresponding set $\bigcap_{i=1}^{\infty} P_{i}$ is the example sought ( because the sets $\bigcap_{i=1}^{\infty} P_{i}$ and $\bigcap_{i=1}^{\infty} P_{i}^{\prime}$ corresponding to different increasing sequences of prime numbers $\alpha=\left\{q_{1}, q_{2}, q_{3}, \ldots\right\}$ and $\alpha^{\prime}=\left\{q_{1}^{\prime}, q_{2}^{\prime}, q_{3}^{\prime}, \ldots\right\}$ are distinct: if $q_{l} \in \alpha \backslash \alpha^{\prime}$ then $\left.x^{c_{l}} \in \bigcup_{i=1}^{\infty}\left\langle x^{c_{i}}\right\rangle \backslash \bigcup_{j=1}^{\infty}\left\langle x^{c_{j}^{\prime}}\right\rangle\right)$. 


\section{Acknowledgements}

The author is grateful to his advisor Professor A.Yu. Ol'shanskii for suggesting problems, helpful discussions and comments.

\section{References}

[1] H.B. Short, Quasiconvexity and a Theorem of Howson's, Group Theory from a Geometrical Viewpoint, Proc. ICTP Trieste, Word Scientific, Singapore, 1991.

[2] Notes on word hyperbolic groups, ed. H.B. Short, Group Theory from a Geometrical Viewpoint, Proc. ICTP Trieste, Word Scientific, Singapore, 1991.

[3] A. Yu. Ol'shanskii, Periodic Quotiens of Hyperbolic Groups, Mat. Zbornik 182 (4), 1991, 543-567.

[4] M. Gromov, Hyperbolic groups, Essays in Group Theory, ed. S.M.Gersten, Math. Sci. Res. Inst. Publ. , Vol. 8 , Springer , 1987, pp. 75-263.

[5] A.Yu. Ol'shanskii, On residualing homomorphisms and G-subgroups of hyperbolic groups, International Journal of Algebra and Computation, Vol.3 , No. 4 (1993), pp. 365-409.

[6] E. Ghys and P. de la Harpe, Sur les Groupes Hyperboliques d'après Michael Gromov, Progress in Mathematics, Vol.83, Birkhauser, 1990.

[7] M. Coornaert, T. Delzant, A. Papadopolous, Notes sur les groupes hyperboliques de Gromov, Lecture Notes in Mathematics, Vol. 1441, Springer, 1990.

[8] G.N. Arzhantseva, On quasiconvex subgroups of word hyperbolic groups, Geometriae Dedicata 87, 2001, no. 1-3, 191-208.

[9] M. Mihalik, W. Towle, Quasiconvex subgroups of negatively curved groups, Pure and Applied Algebra, 95 (1994), 297-301.

[10] S.M. Gersten and H. Short, Rational subgroups of biautomatic groups, Annals of Math. 134 (1991), 125-158.

[11] Zeph Grunschlag, Computing angles in hyperbolic groups, Groups, Languages and Geometry, R. Gilman Ed., Contemporary Math. 250 (1999), 59-88.

[12] E. Rips, Subgroups of small cancellation groups, Bull. London Math. Soc., 14 (1982), No. 1, 45-47. 